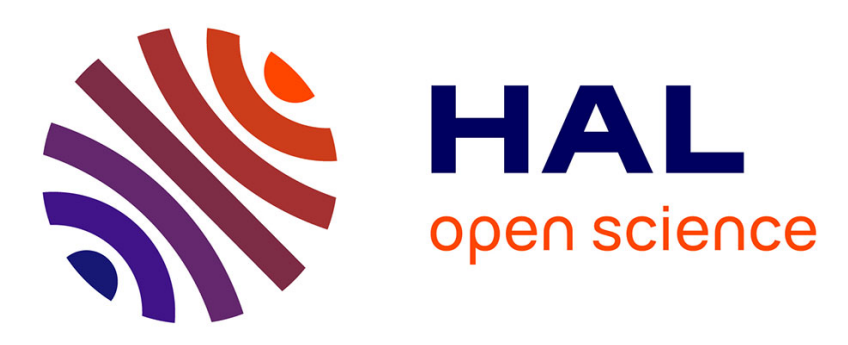

\title{
Action Observation Network activity related to object-directed and socially-directed actions in Adolescents
}

Mathieu Lesourd, Alia Afyouni, Franziska Geringswald, Fabien A Cignetti, Lisa Raoul, Julien Sein, Bruno Nazarian, Jean-Luc Anton, Marie-Hélène Grosbras

\section{To cite this version:}

Mathieu Lesourd, Alia Afyouni, Franziska Geringswald, Fabien A Cignetti, Lisa Raoul, et al.. Action Observation Network activity related to object-directed and socially-directed actions in Adolescents. 2020. hal-03084473

\author{
HAL Id: hal-03084473 \\ https://hal.science/hal-03084473
}

Preprint submitted on 21 Dec 2020

HAL is a multi-disciplinary open access archive for the deposit and dissemination of scientific research documents, whether they are published or not. The documents may come from teaching and research institutions in France or abroad, or from public or private research centers.
L'archive ouverte pluridisciplinaire HAL, est destinée au dépôt et à la diffusion de documents scientifiques de niveau recherche, publiés ou non, émanant des établissements d'enseignement et de recherche français ou étrangers, des laboratoires publics ou privés. 


\section{Action Observation Network activity related to object-directed and}

3 Running title : Action Observation Network in Adolescents

4

Mathieu Lesourd ${ }^{1}$, Alia Afyouni ${ }^{2}$, Franziska Geringswald ${ }^{2}$, Fabien Cignetti ${ }^{3}$, Lisa Raoul ${ }^{2}$,

Julien Sein ${ }^{4}$, Bruno Nazarian ${ }^{4}$, Jean-Luc Anton ${ }^{4}, \&$ Marie-Hélène Grosbras ${ }^{2}$

${ }^{1}$ Laboratoire de Psychologie (EA 3188), Université de Bourgogne Franche-Comté, Besançon,

9 France

${ }^{2}$ Aix Marseille Univ, CNRS, LNC, Laboratoire de Neurosciences Cognitives, Marseille,

\section{France}

$12{ }^{3}$ Univ. Grenoble Alpes, CNRS, TIMC-IMAG, F-38000 Grenoble, France

$13{ }^{4}$ Aix Marseille Univ, CNRS, Centre IRM-INT@CERIMED (Institut des Neurosciences de la 14 Timone - UMR 7289), Marseille, France

Corresponding authors email address:

mathieu.lesourd@univ-fcomte.fr

18 marie-helene.grosbras@univ-amu.fr

Manuscript page number: 45 pages

Number of figures/tables: 7 Figures and 1 Table

Word count

- Abstract: 249 words

- Introduction: 638 words

- Discussion: 1442 words

Acknowledgements: This research was supported by grants from the Agence Nationale de la

Conflict of interest: The authors declare no competing financial interests 


\section{Abstract}

The Action Observation Network (AON) encompasses brain areas consistently engaged when we observe other's actions. Although the core nodes of the AON are present from childhood, it is not known to what extent they are sensitive to different action features during development. As social cognitive abilities continue to mature during adolescence, the AON response to socially-oriented actions, but not to object-related actions, may differ in adolescents and adults. To test this hypothesis, we scanned with functional magnetic resonance imaging (fMRI) 28 typically-developing teenagers and 25 adults while they passively watched videos of hand actions varying along two dimensions: sociality (i.e. directed towards another person or not) and transitivity (i.e. involving an object or not). We found that observing actions recruited the same fronto-parietal and occipito-temporal regions in adults and adolescents. The modulation of voxelwise activity by the social or transitive nature of the action was similar in both groups of participants. Multivariate pattern analysis, however, revealed that the accuracy in decoding the social dimension from the brain activity patterns, increased with age in lateral occipital and parietal regions, known to be involved in semantic representations of actions, as well as in posterior superior temporal sulcus, a region commonly associated with perception of high level features necessary for social perception. Change in decoding the transitive dimension was observed only in the latter region. These findings indicate that the representation of others' actions, and in particular their social dimensions, in the adolescent $\mathrm{AON}$ is still not as robust as in adults.

Keywords: Action Observation; Adolescence; fMRI; Social actions; Object-direct actions 


\section{Significance statement}

58 The activity of the action observation network in the human brain is modulated according to

59 the purpose of the observed action, in particular the extent to which it involves interaction

60 with an object or another person. How this conceptual representation of actions is

61 implemented during development is largely unknown. Here, using multivoxel pattern analysis

62 of fMRI data, we discovered that, while the action observation network is in place in

63 adolescence, the fine-grain organization of its posterior regions is less robust than in adults to

64 decode the social or transitive dimensions of an action. This finding highlights the late

65 maturation of social processing in the human brain.

66

67 


\section{Introduction}

When we observe other's actions a set of brain areas is consistently engaged contributing to our social interactions' capability. The so-called Action Observation Network (AON) comprises fronto-parietal regions -- traditionally associated with action planning (Gallese et al., 1996; Buccino et al., 2001) - as well as posterior superior temporal sulcus (pSTS) and high-level visual occipito-temporal areas -- traditionally associated with perceptual analyses (Carr, Iacoboni, Dubeau, Mazziotta, \& Lenzi, 2003; Downing, 2001; for meta-analyses see Caspers, Zilles, Laird, \& Eickhoff, 2010; Grosbras, Beaton, \& Eickhoff, 2012) ADD OSTENHORF. The AON supports not only the representation of low-level aspects of an action (e.g., kinematics) but also its high-level aspects (e.g., goal, intention) indexing the abstract or conceptual knowledge about the action observed [WURM; LIGNAU; HAFRI; URGEN]. Notably, a number of empirical and theoretical studies suggest that activity in different subsystems of the AON might be modulated by the social aspect of the observed actions, that is whether they involve another agent or not. For instance, watching point-light displays representing two individuals interacting enhanced the recruitment of the inferior frontal gyrus (IFG), premotor areas, bilateral IPS and the right superior parietal lobe (SPL), as compared to watching the same individuals acting independently (Centelles et al., 2011). Higher activity in fronto-parietal (Oberman et al., 2007; Becchio et al., 2012) and occipitotemporal parts (Saggar et al., 2014; Isik et al., 2017; Wurm et al., 2017; Walbrin et al., 2018; Becchio et al., 2012) of the AON has also been reported when participants observed gestures or object-directed actions performed with a social intent (e.g. communicating or cooperating) as compared to individual-centered actions. Multivoxel patterns and representation similarity analyses have revealed a representation of both the objectdirectedness (transitivity) and person directedness (sociality) qualification of an action in most part of the AON. Yet results seems to converge to indicate that only in the posterior part 
these representations, and in particular the social representation, generalize well across a variety of perceptually divergent actions (Wurm and Caramazza, 2019; but see Hafri 2017), and even verbal description (Wurm and Caramazza Nat Communication 19.

The general notion of the existence of a conceptual representation of action content and in particular the social orientation of an action, raises the question of the ontogeny of this representation. This question is even more complex given the late maturation of this part of the brain during adolescence, both in terms of structure (e.g. grey matter density) and functional connectivity patterns, in contrast to premotor regions that seem to mature earlier. HYP If shaped by experience fine grained representation should change during adolescence. Responses of parts of the AON are present very early in development. Activity during passive observation of other people's hand actions has been reported in sensorimotor (Shimada and Hiraki, 2006) and temporal areas (Lloyd-Fox et al., 2009) in 5-months old infants using near-infrared spectroscopy. Functional magnetic resonance imaging (fMRI) studies in children (from 7 years old) and adolescents showed that all nodes of the AON are identified during action observation (Ohnishi et al., 2004; Shaw et al., 2011, 2012; Pokorny et al., 2015). Direct comparison with adults showed a lower activity in occipito-temporal areas in children age 7-9 (Morales et al., 2019) and less left-lateralization in occipital regions in a group of 7 to 15 years old (Biagi et al., 2016). So far, however, no study has investigated whether the representation of the content of observed actions is the same as in adults.

Yet social perception skills continue to mature especially during adolescence (Scherf et al., 2007; Ross et al., 2014) while social orientation and social cognition also undergo a drastic increase in complexity (Steinberg and Morris, 2001). Besides, structural changes in the AON regions still occur until the end of the teenage years, which suggests also changes in functional organization (Mills et al., 2014). In these regards, we hypothesized that the modulation of the action observation network when the type of action is of social nature might also change. We 
118 designed an fMRI paradigm where adolescents (13-17 years old) and adults passively watched

119 short videos of actions that varied in their social or transitive nature. We asked specifically

120 whether, at an age when the overall activity of the AON is adult-like, the local representations

121 of the different conceptual dimensions of action is also already mature. In line with the delayed

122 development of social cognition, we would expect bigger differences within the AON between

123 adolescents and adults for social actions only.

124

\section{Material and Methods}

\subsection{Participants}

Twenty-eight typically developing adolescents aged from 13 to 17 years $\left(M_{\text {age }}=15.1, S D\right.$ $=1.26 ; 13$ females; 27 right-handers) were enrolled in the study. They completed the Pubertal Development Scale (PDS; Petersen \& Crockett, 1988), a sex-specific eight-item self-report measure of physical development based on Tanner stages (Marshall \& Tanner, 1969, 1970). Adolescents answered questions concerning their physical development (e.g. growth in stature, breast development, pubic hair) and on the basis of their answers they were assigned to one of the categories of pubertal status: mid-pubertal (Tanner stage $3, n=9$ ), advanced pubertal (stage 4, $n=13$ ), and post-pubertal (stage 5, $n=6$ ). Twenty-five adults $\left(M_{\text {age }}=26.6\right.$, $S D=2.02$, range $=24-33$ years old; 14 females; 22 right-handers) were also recruited in the study. Recruitment was made through internal ads in the university.

All participants reported to be healthy and typically developing, they had normal or corrected-to-normal vision and reported no history of neurological or psychiatric disorder. All participants were voluntary and signed written consent. Written consent was also obtained from the adolescents' parents. The study was in line with the Declaration of Helsinki and was approved by the national Ethics Committee. 
Inclusion in the final sample required that head motion during scanning did not exceed $2 \mathrm{~mm}$ displacement between consecutive volumes on $90 \%$ of volumes for each run. One male adolescent was excluded based on this criterion. One adult was also excluded following

144 technical problems during fMRI scanning.

\subsection{Stimuli}

The stimuli consisted of 256 videos, each representing the same scene with two persons, amongst four possible actors, facing each other across a table, seen from the side (i.e. one actor on each side of the screen). Only the arms and hands of the actors were visible.

Different objects were placed on the table. Only one of the two actors produced an action with her/his right or left arm. There were no physical contact between the two actors.

We grouped the actions into four classes, based on whether the action depicted involved the other person or not (Social or Non-Social) and whether it involved an object or not

153 (Transitive or Intransitive). We had 64 exemplars of videos for each class that represented the following actions: (1) Social Transitive (ST): give/take pen and give/take book; (2) NonSocial Transitive (NT): write/rub with pencil and open/close book; (3) Social Intransitive (SI): agree/disagree finger gesture and come/go away hand gesture ; and (4) Non-Social Intransitive (NI): stroke/scratch arm with finger and stroke/scratch arm with hand. actor sitting on the left or right side of the table and filmed from two slightly different

160 perspectives. This maximized chances to identify representational mechanisms that rely on 161 abstract action representations that generalize across perceptual information (Wurm, Ariani,

162 Greenlee, \& Lingnau, 2016; Wurm, Caramazza, \& Lingnau, 2017; Wurm \& Lingnau, 2015). In addition, we added control items consisting of eight modified action videos from

164 the four action classes ( 2 control videos per action class). In these videos, the actors were 
165 removed, and a pink disk moved within the scene. The trajectory and cinematic of the disk were matched with that of the gesture from the original video.

169 player (available from $\underline{\mathrm{https}} / / \mathrm{mpv} . \mathrm{io} /$ ) to determine the onset and duration of each action.

170 Individual action duration was then standardized across action class by slightly speeding up or

171 slowing down the individual videos for which the duration of the action fell outside the mean +/- two times the standard deviation of all videos of the respective action class. A variable number of 'filler' frames before and after the execution of the action were included for each

174 video to create final trial videos, consisting of a combination of three videos of the same

175 action class each (see below), of equal length. All video editing was performed using ffmpeg

176 (version 3.2, available from http://ffmpeg.org/) and in-house Python scripts. The quantity and

177 spatial amplitude of motion was inevitably different for each class of action. For instance, the

178 social action "Thumb down" implies a large gesture of the arm whereas the non-social action

179 "Scratch" implies a local gesture with low arm amplitude. As a consequence, the global and

180 local visual motion was different across classes. In order to quantify and control in subsequent analyses for potentials effects of these interclass differences, we used a program developed in-

182 house in Python with the library OpenCV (Open Source Computer Vision Library;

183 https://opencv.org/) to compute, for each video frame, the number of pixels that changed

184 intensity relative to the preceding frame. Then, the total number of changing pixels was

185 divided by the total number of frames to obtain a score of motion magnitude. Videos of social

186 actions involved more visual motion than videos of non-social actions. We thus used the

187 motion magnitude score as a regressor of non-interest in the analysis of brain activity (see

188 section Univariate fMRI Analysis, for more details). 

were asked to rate each video using visual analog scales (from $0=$ not at all to $100=$ very much), along two dimensions introduced with the following questions: for sociality, "How much is the action relevant for the nonacting person?"; for transitivity, "How much does the action involve the interaction with a physical object?". As expected, the four categories were transitive actions.

For the fMRI experiment, to maximize the BOLD response elicited by each action observation condition, videos were arranged in triplets that varied across the identity of actors, the perspective, and the side of action. This resulted in $9.5 \mathrm{~s}$ videos showing the same action class, hereafter called trial videos, that were used in a block design.

\section{3. fMRI experiment}

Each participant was scanned in a single-session with: (i) a T1-weighted anatomical scan, (ii) one practice functional run to ensure that participants felt comfortable with the task, (iii) eight functional runs. Each functional run contained 20 trials (16 action trials plus 4 control

$3 \mathrm{~s})$ followed by a trial video $(9.5 \mathrm{~s})$, which was then immediately followed by a blank screen

209 (variable duration from 0.5 to $1.5 \mathrm{~s}$ ) and a subsequent rating screen $(5 \mathrm{~s})$. The inter-trial-

210 interval thus varied from $16.12 \mathrm{~s}$ to $19.12 \mathrm{~s}$. Each run ended with a $10 \mathrm{~s}$ fixation period. A

211 genetic algorithm was used to optimize the experimental design with regards to contrast

212 estimation (Wager and Nichols, 2003; Kao et al., 2009) using the toolbox NeuroDesign

213 (https://neurodesign.readthedocs.io/en/latest/index.html). We thereby created eight different 
214 schedules of sequences of conditions and intertrial intervals. The assignment of these

215 schedules to the eight runs was counterbalanced across participants. pixels screen resolution) via a liquid crystal DLP projector (OC EMP 7900, Epson) and viewed through a mirror mounted on the head coil. Image on the screen had a 40x30 $\mathrm{cm}$ size, covering a $20^{\circ}$ angle of view. Participants gaze position on the projection mirror was recorded (Eyelink 1000 system, SR Research). Before each functional run, the spatial accuracy of the calibration of the eye tracker was validated using 9 points. If the average deviation exceeded $1^{\circ}$ of visual angle, the spatial calibration was redone. Stimulus presentation, response collection and synchronization with the fMRI acquisition triggers and the eyetracker were implemented in a custom-built program, using the LabVIEW (National Instrument) environment. After each functional run, participants were allowed self-determined breaks.

Participants were first asked to watch attentively each trial video. Immediately after a trial video, a response screen, showing a question and a slider, was presented and participants 230 had to indicate, depending on the question, either the degree of sociality or the degree of transitivity of the action that was depicted in all the three videos they had just seen. We used

232 the same questions as in the preliminary independent experiment. Participants gave their response by moving a track-ball with their right index along an analog-scale (from $0=$ not at

234 all to $100=$ very much) and validated their choice by clicking with their right thumb. Only one question was displayed for each trial. As a trial video was presented twice during the experiment, both social and transitive ratings were collected for each action. The order of

237 presentation of the questions was counterbalanced across subjects. Ratings were used to 
transitivity. Importantly, as participants did not know in advance which question would be

240 asked, they were not biased towards attending to one or the other dimension. Two questions

241 were also asked for the control videos, one concerning the distance covered and the other

242 concerning the velocity of the pink disk. To ensure that participants understood and followed

243 correctly the instructions during the fMRI session, they completed a practice run before the

244 scanning, outside the scanner. No information about the exact aim of the study was given

245 before the experiment.

\subsection{Data acquisition}

Imaging data were acquired on a 3T Siemens Prisma Scanner (Siemens, Erlangen,

248 Germany) using a 64-channel head coil. Blood-Oxygen Level Dependent (BOLD) images

249 were recorded with T2*-weighted echo-planar images acquired with the multi-band sequence

250 (version R016a for Syngo VE11B) provided by the University of Minnesota Center for

251 Magnetic Resonance Research (https://www.cmrr.umn.edu/multiband/). Functional images

252 were all collected as oblique-axial scans aligned with the anterior commissure-posterior

253 commissure (AC-PC) line with the following parameters: 287 volumes per run, 54 slices,

$254 \mathrm{TR} / \mathrm{TE}=1224 \mathrm{~ms} / 30 \mathrm{~ms}$, flip angle $=66^{\circ}$, field of view $=210 \times 210 \mathrm{~mm}^{2}$, slice thickness $=$

$2552.5 \mathrm{~mm}$, voxel size $=2.5 \times 2.5 \times 2.5 \mathrm{~mm}^{3}$, multiband factor $=3$. To correct for magnetic field

256 inhomogeneity during data preprocessing, we also acquired a pair of spin-echo images with

257 reversed phase encoding direction $\left(\mathrm{TR} / \mathrm{TE}=7.060 \mathrm{~ms} / 59 \mathrm{~ms}\right.$, flip angle $=90^{\circ}$, voxel size $=$

$2582.5 \times 2.5 \times 2.5 \mathrm{~mm}^{3}$ ). Structural T1-weighted images were collected using a $\mathrm{T} 1$ weighted

259 Magnetization-Prepared 2 Rapid Acquisition Gradient Echoes (MP2RAGE) sequence (176

260 sagittal slices, TR/TE $=5000 / 2.98 \mathrm{~ms}, \mathrm{TI} 1 / \mathrm{TI} 2=757 / 2500 \mathrm{~ms}$, alpha1 $/$ alpha $2=4^{\circ} / 5^{\circ}$,

261 Bandwidth $=240 \mathrm{~Hz} /$ pix, Field-Of-View $=256 \times 256 \times 176 \mathrm{~mm}^{3}$, slice thickness $=1 \mathrm{~mm}$,

262 voxel size $=1 \times 1 \times 1 \mathrm{~mm}^{3}$ ). 


\subsection{Preprocessing}

Structural T1-weighted images were derived from MP2RAGE images by removing the noisy background and were skullstripped and segmented into tissue type (GM: grey matter, WM: white matter and CSF: cerebro-spinal fluid tissues) using the Computational Anatomy Toolbox (CAT12; http://dbm.neuro.uni-jena.de/cat12/). Functional data were analyzed using

SPM12 (Wellcome Department of Cognitive Neurology, http://www.fil.ion.ucl.ac.uk/spm) implemented in MATLAB (Mathworks, Sherborn, MA). Preprocessing for univariate analyses included the following steps (1) realignment to the mean EPI image with 6-head motion correction parameters; (2) co-registration of the individual functional and anatomical were skipped to work only on unsmoothed EPI images, in native space of each subject.

279 (with onsets and durations corresponding to the start of each video of that condition)

280 convolved with the canonical hemodynamic response function (HRF) of SPM, one regressor

281 for the control condition, built the same way, one regressor accounting for judgement and

282 motor response (HRF-convolved boxcar function containing all the periods during which the rating screen was presented and responses given) and six regressors of non-interest resulting

284 from 3D head motion estimation ( $\mathrm{x}, \mathrm{y}, \mathrm{z}$ translation and three axis of rotation). As quantity 285 and spatial amplitude of visual motion was different for each class of action, we also included one regressor controlling for unequal motion quantity. This regressor was modeled as a 
and parametrically modulated with motion quantity values (z-scored for each run). A

regressor accounting for eye movements was also included with each saccade modeled

toolbox (Kasper et al., 2017). We extracted the time-course of the signal from all voxels in the

293 CSF and separately in the white matter. A principal component analysis (PCA) was

294 performed (i.e., CompCor; Behzadi et al., 2007), and fourteen physiological components

related to non-BOLD activity were extrapolated in the normalized WM (6 first PCs + mean participant, also taking into account the average signal in each run. The contrast of parameter estimates of each condition compared to control, computed at the individual level, were entered into a three-way repeated measures ANOVA, with Group (Adolescents vs Adults) as between-subject factor, and Sociality (social vs non-social) and Transitivity (transitive vs intransitive) as within-subject factors. The analysis was performed using GLMflex (http://mrtools.mgh.harvard.edu/index.php/GLM_Flex) implemented in Matlab. We present results maps with a significance threshold set at $p_{\mathrm{FWE}}<.05$ with family-wise error (FWE) correction applied at the cluster level (cluster-defining non-corrected threshold at $p<.001$ ).

\subsection{Multi-voxel pattern analysis}

\subsubsection{Regions of interest (ROI) definition}

In a first analysis, we focused on regions typically recruited during action observation. We

311 These ROIs were derived from an independent meta-analysis of fMRI and PET data

312 (Grosbras et al., 2012), by taking the conjunction of activated voxels reported in a set of 
313 studies contrasting observing hand movements (with or without object) to control conditions

314 ( $p<.001$ uncorrected, cluster extent threshold of 5 voxels).

315 All ROIs, defined in MNI-space, were transformed into each subject native space and

316 masked with his grey matter mask. Importantly, overlapping voxels across ROIs were

317 manually inspected and were attributed to the smallest ROI to ensure all ROIs were

318 independent of each other (Bracci et al., 2017). This concerned only left pSTS and left LOTC

319 due to their spatial proximity in the meta-analysis results and represents a marginal number of

320 voxels $(M=6.51 \pm 2.68)$.

Each ROI had a different number of voxels across subjects and hemispheres (mean size

and standard deviation are indicated in brackets): LOTC (left $=236.45 \pm 33.60$, right $=316.57$

$\pm 51.57), \operatorname{PMv}($ left $=139.57 \pm 23.15$, right $=143.06 \pm 24.77), \operatorname{pSTS}($ left $=71.84 \pm 13.20$

right $=75.43 \pm 17.98)$, and IPS/SPL $($ left $=213.04 \pm 37.92$, right $=171.92 \pm 27.93)$. These

differences may prevent the reliability of between subjects' comparisons and may bias group procedure, separately for each classification analysis, based on the highest values in the univariate F-test. Thereby for each subject we defined ROIs with size constrained by the smallest size observed across participants in the initial definition: LOTC [ $n=174$ voxels], PMv $[n=96$ voxels], pSTS [ $n=47$ voxels], and IPS/SPL [ $n=104$ voxels $]$.

\subsubsection{ROI-based MVPA}

We performed multivoxel pattern analyses (MVPA) within the eight ROIs independently. At the individual level we computed a new GLM using the realigned and unwarped images in native space (without smoothing) and estimating single trial activity (i.e. using 20 regressors 
338 (Abraham et al., 2014) for Python 3.7. For voxels within each ROI, we trained, on a subset of

339 data, a linear support vector machine classification (regularization hyperparameter $\mathrm{C}=1$ ), to

340 distinguish patterns of parameter estimates associated with each condition. We then tested the

341 classifier ability to decode the conditions associated with patterns of parameter estimates on

342 the remaining data. We used an eight-fold leave-one out cross-validation schedule, training on

343 data from seven runs and testing on data from the remaining run and averaging the

344 classification accuracies (percent correct) across the eight iterations.

345 This procedure was carried out independently in each ROI in two analyses: firstly, we

346 trained the classifier to discriminate social versus non-social actions (112 patterns from the

347 seven runs of the training set: 56 social and 56 non-social), independently of the transitive

348 dimension, and we tested on the remaining 16 patterns (8 social and 8 non-social); secondly,

349 we trained the classifier to discriminate transitive versus intransitive actions (112 patterns: 56

350 transitive and 56 intransitive), independently of the social dimension, and we tested on the

351 remaining 16 patterns ( 8 transitive and 8 intransitive) (see Figure 2). For each analysis, to

352 make group-level inferences we compared the averaged accuracies per ROI to chance level

$353(50 \%)$ using a one-tailed one-sample Student $t$-test. Statistical results were FDR-corrected for

354 the number of ROIs (Benjamini and Yekutieli, 2001). We also assessed the significance of

355 decoding at the individual level with a fold-wise permutation scheme (Etzel and Braver,

356 2013). To do so, the classification was repeated 1000 times after randomizing the labels in

357 order to construct a null-distribution per subject, ROI and condition. The $p$-value was then

358 given by dividing the number of times where the mean classification accuracy was greater

359 than the classification score obtained by permuting labels, by the number of permutations.

360 We entered classification accuracies in a two-way ANOVA with Age group (adolescents

361 and adults) as between factor and Hemisphere (Left and Right) as within factor. 

matrices to explore which conditions might be confounded to each other. Mean accuracies for each action class (values in the confusion matrix diagonal) was then entered in ANOVAs to significant effect (i.e. above chance global decoding of action category) was found.

\subsubsection{Searchlight MVPA}

To complete the results obtained with the ROI-based decoding and test the presence of additional putative brain areas for decoding Social vs Non-Social and Transitive vs Intransitive, we carried out a whole-brain searchlight analysis with $12 \mathrm{~mm}$ radius spheres (about 463 voxels). MVPA classification was carried out with the same parameters and procedure as the ROI-based MVPA, within each sphere as the searchlight moved across the brain, and the classification accuracy was stored at the central voxel, yielding a 3D brain map of classification accuracy (Haynes, 2015). To identify regions where classification accuracy was significantly above chance (i.e., 50\%) in adults and adolescents, the chance level was subtracted from classification maps, then these maps were normalized (MNI template) and smoothed $(\mathrm{FWHM}=6 \mathrm{~mm})$. Then, we carried out one-sample t-tests for each group and each condition separately, corrected for multiple comparisons at the cluster level (FWE, $p<.05)$.

\section{Results}

\subsection{Behavioral ratings}

We carried out one three-way ANOVA (Group x Sociality x Transitivity) separately for each rating (i.e., sociality and transitivity). Concerning the rating of the transitive dimension, we found a main effect of Transitivity $F(1,49)=176.88, p<.001, \eta_{p}{ }^{2}=.97$, transitive actions 
$387(M=.93, S D=.32)$ were rated more transitive than intransitive videos $(M=-.93, S D=.24)$, unsurprisingly. No other main effect nor interaction including the factor group were found. Concerning the rating of the social dimension, we found a main effect of Sociality $F(1,49)=$ $623.82, p<.001, \eta_{p}{ }^{2}=.93$, social videos $(M=.79, S D=.51)$ were rated more social than the non-social actions $(M=-.79, S D=.49)$. We also found a main effect of Transitivity $F(1,49)=$ transitive actions $(M=-.14, S D=.71)$. Finally, the ANOVA revealed an interaction between

394 Sociality and Transitivity $F(1,49)=42.50, p<.001, \eta_{p}{ }^{2}=.46$ : there was no difference between non-social transitive $(M=-.72, \mathrm{SD}=.38)$ and non-social intransitive actions $(M=$ $.86, \mathrm{SD}=.58, p=.47)$, whereas social intransitive actions $(M=1.14, \mathrm{SD}=.29)$ were rated more social than social transitive videos $(M=.44, \mathrm{SD}=.44, p<.001)$. No other main effect nor interaction including the factor group were significant.

\subsection{Univariate fMRI results}

We entered the individual maps of parameters estimates for the four action conditions (NI, NT, SI, ST) in a repeated-measure ANOVA with Sociality and Transitivity as within-subject factors and Age group as between-subject factor. The results are displayed in Table 1 and

Figure 3. compared to non-social actions induced stronger activity in AON regions in bilateral posterior 408 superior temporal sulcus and bilateral middle temporal gyrus, bilateral supramarginal gyrus, bilateral precentral gyrus, in left superior parietal lobe and in left inferior frontal gyrus

410 bilateral, as well as in superior frontal gyrus, SMA, precuneus bilateral visual cortices

411 (intracalcarine cortex and lingual gyrus). The reverse contrast yielded significant activation in 
412 left anterior parietal cortex (AIPS/SPL), left inferior occipital cortex and right precentral

413 gyrus, as well as in occipital pole and lateral occipital cortex.

414 We found a main effect of Transitivity (see Figure 3B): observing transitive actions was

415 associated with stronger activity in bilateral medial occipital cortex, bilateral precentral

416 cortex, right superior frontal sulcus, left parieto-occipital cortex, right inferior temporal

417 cortex, bilateral cerebellum (lobule VIII/IX), left angular gyrus and right posterior cingulate

418 cortex. The reverse contrast revealed significant activations in bilateral early visual cortices

419 (cuneus), right lateral occipital temporal cortex (EBA/FBA), right posterior superior temporal

420 cortex (SMG/pSTS), bilateral temporal poles, right pericentral cortex (central sulcus and

421 postcentral cortex).

There was also a main effect of Age group. The contrast adolescents versus adults

revealed higher activation in adolescents, when observing action compared to the control

condition activation, in left ventral medial prefrontal cortex and in left temporoparietal

junction (Figure 3C).

We did not observe any significant interaction between Sociality and Transitivity in any region. Finally, the ANOVA did not reveal any interaction between the factors Sociality or

428 Transitivity and Age group nor three-way interaction.

\subsection{ROI MVPA}

\subsubsection{Decoding social vs non-social and transitive vs intransitive actions}

Significant above-chance decoding was found in all the regions of the AON, for both

adolescents and adults. LOTC and IPS/SPL showed the highest decoding accuracies for the

433 social dimension and LOTC for the transitive dimension (Figure 4). We also assessed the

434 significance of decoding in these regions at the individual level using permutations (Etzel \&

435 Braver, 2013; see Extended Data Figure 4-1), with a cutoff of $p<.05$. For the social

436 dimension, all adults (left $=100 \%$ and right $=100 \%)$ and nearly all adolescents $(\mathrm{left}=93 \%$ 
and right $=96 \%$ ) decoded significantly in the LOTC, but in IPS/SPL the proportion of

438 adolescents (left $=70 \%$ and right $=44 \%$ ), for whom decoding was significant, was lower than

439 that of adults (left $=79 \%$ and right $=79 \%)$. For the transitive dimension, decoding was

440 significant in all participants in the LOTC.

In a second step, we compared classification performance for adolescents and adults in

443 LOTC, PMv, pSTS, and IPS/SPL, by entering mean classification accuracies in two-way

444 ANOVAs with Hemisphere (Left, Right) as within subject factor and Age group

445 (Adolescents, Adults) as between factor. These analyzes were performed for each dimension

446 (i.e., transitivity and sociality) separately. Concerning the social dimension, the ANOVAs

447 revealed a main effect of Age group in IPS/SPL, $F(1,49)=9.2, p<.01$, in pSTS, $F(1,49)=$

$4488.17, p<.01$, and in LOTC, $F(1,49)=7.23, p<.01$, with higher decoding values for adults.

449 There was a main effect of Hemisphere in LOTC, $F(1,49)=9.11, p<.01$, and in IPS/SPL,

$450 F(1,49)=4.07, p=.049$, with higher decoding values in the right hemisphere. There was no

451 interaction between Hemisphere and Age group (All $p>.10$ ). Concerning the transitive

452 dimension, the ANOVAs revealed a main effect of Age group in pSTS, $F(1,49)=6.35, p=$

453.015 . There was a main effect of Hemisphere in $\operatorname{pSTS}, F(1,49)=16.64, p<.001$. There was

454 no interaction between Hemisphere and Age group (All $p>.10)$.

\subsubsection{Searchlight MVPA}

Significant decoding was found for social and transitive actions bilaterally in brain areas typically associated with the AON including LOTC, PMv, pSTS, and IPS/SPL in both groups 458 of participants (see Figure 5A). Moreover, when comparing accuracy maps for adults and 459 adolescents using two-sample t-tests, we found significant clusters in bilateral IPS for Social 460 versus Non-Social actions and in right pSTS for Transitive vs Intransitive actions (see Figure

461 5B and Extended Data Table 5-1), thus confirming the results obtained in the ROI analysis. 


\subsubsection{Decoding individual action classes (NI, NT, SI, and ST)}

464

465

466

467

468

469

470

471

472

473

474

475

476

477

478

479

480

481

482

483

484

485
We also carried out a four-way classification in each ROI and each participant and derived confusion matrices representing the pairwise decoding accuracies across conditions (i.e. how often a pattern corresponding to a condition is correctly decoded: matrix diagonal) and confounded with each of the other conditions (see Figure 6). The classifier was able to correctly discriminate each action class above chance in LOTC, IPS/SPL, and PMv and in a lesser extent in pSTS (see Extended Data Figure 6-1). The confusion matrices were highly similar between adults and adolescents.

$<$ Insert Figure 6 about here $>$

To investigate potential differences between adolescents and adults for each action class, mean classification accuracies were entered in ANOVAs with Sociality and Transitivity as within-subject factor and Age group as between-subject factor. Mean classification accuracies were averaged from the two hemispheres, as no interaction with the factor Hemisphere was significant in the first ROI MVPA. We carried out ANOVAs separately for each ROI (LOTC, PMV, IPS/SPL, and pSTS). These analyzes revealed a trend to significance for the interaction Age Group x Sociality x Transitivity in the IPS/SPL, $F(1,49)=3.90, p=0.054$ (Figure 7A), but this double interaction was not significant either in the LOTC, $F(1,49)<1$, in PMv, $F(1,49)<1$, or in the pSTS, $F(1,49)<1$. In the IPS/SPL, decoding accuracies were higher for adults compared to adolescents for NT, $t(49)=-2.10, p=.02$, SI, $t(49)=-2.41, p<.01$, and ST, $t(49)=-1.71, p=.047$, but not for NI, $t(49)=-.51, p=.31$. Finally, we found a significant correlation between decoding accuracies and chronological age in IPS/SPL only in adolescents for SI, $r(25)=.47, \mathrm{p}=.012$, and ST, $r(25)=.52, \mathrm{p}<.01$ (see Figure 7B).

$<$ Insert Figure 7 about here $>$ 


\section{Discussion}

Our univariate analyses indicate that all components of the AON are in place in adolescence and are engaged to the same level as in adults. Moreover, multivariate analyses showed that, like in adults, regions of this network contain information related to the content of actions. Yet this fine-grained action representation becomes more robust between adolescence and adulthood in IPS/SPL, pSTS and LOTC. Additionally, outside the AON we observed higher activity in adolescents in the MPFC and TPJ, two regions of the mentalizing network. and early adolescence (Ohnishi et al., 2004; Pokorny et al., 2015; Biagi et al., 2016; Morales et al., 2019) by testing advanced and post pubertal adolescents (14-17 years old).

497 Furthermore, we show that the modulation of AON activity by the transitive and social 498 dimensions of the observed actions is similar in adolescents and adults. Social actions induced 499 higher activity than non-social actions in the pSTS, supramarginal gyrus, and precentral 500 cortex, independently of whether these actions also involved an object. This complements 501 previous adults studies that investigated either object-directed actions with a social intent or

502 communicative symbolic actions or interactions (Iacoboni et al., 2004; Montgomery et al., 503 2007; Centelles et al., 2011; Saggar et al., 2014; Sliwa and Freiwald, 2017; Walbrin et al., 2018). In contrast, non-social actions engaged the most posterior parts of the temporal occipital cortex, as well as anterior parietal/post central areas, perhaps in relation to the fact 506 that they drew attention to somato-sensation in the active actor, in particular in the stroking or 507 rubbing videos. Observing transitive, relative to intransitive, actions yielded significant 508 activation in bilateral medial fusiform gyrus, which is not typically included in the AON, but 509 involved in processing information about objects (Mahon et al., 2007) and object-directed

510 actions (Chen et al., 2016). We also observed bilateral activation of IPS/SPL and dPMC, 
511 which are part of a frontoparietal network involved in grasping and reaching (Daprati and

512 Sirigu, 2006), as well as in observing others using tools ( rev. in Reynaud, Navarro, Lesourd,

513 \& Osiurak, 2019). Observing intransitive versus transitive actions revealed activation in

514 bilateral pSTS/STS and lateral occipitotemporal cortex (extending into the fusiform gyrus).

515 This latter region is likely to encompass the extrastriate body area (EBA) and the fusiform

516 body area (FBA), which selectively process visual features of human bodies (Downing \&

517 Peelen, 2011). Interestingly, Wagner and colleagues (2016), using naturalistic movie stimuli

518 showed that FMRI signal peaks in the lateral fusiform gyrus occurred more frequently in

519 response to scenes depicting a person (face or body) engaged in a social action, while peaks in

520 the medial fusiform gyrus occurred for scenes with objects, landscapes or buildings,

521 irrespective of the presence of social cues. In line with our data, this suggests that EBA and

522 FBA are more engaged by intransitive than transitive actions stimuli and the reverse for the medial fusiform gyrus.

These findings are comforted by the multivariate analyses that provide evidence of representations of both the social and transitive dimensions of actions in all parts of the AON.

526 Yet, while the univariate analysis did not show any difference between adolescents and

527 adults, multivariate decoding accuracies were lower in adolescents in the LOTC, pSTS and IPS/SPL for social versus non-social actions and in pSTS for transitive versus intransitive 529 actions.

The LOTC contains a mosaic of focal but overlapping regions selective for particular

531 types of information (like hand posture, body shape, tools) that forms the components of

532 action representations important for action understanding and social interpretation (for

533 discussions see Lingnau \& Downing, 2015; Wurm and Caramazza, 2019). Some authors have

534 suggested that the LOTC forms the perceptual anchor of a pathway that extends into the 535 superior temporal cortex and temporal parietal junction, a gradient along which increasingly 
rich representations of the posture, movements, actions, and mental states of other people are constructed (Carter and Huettel, 2013). Here we found higher decoding accuracy in adults only for social but not for transitive actions. This suggests that the role of this region for social action representation is still immature in adolescence.

We also found significant differences between adolescents and adults in representation of the social but not the transitive dimension in a region within the IPS/SPL. This region is

542 part of the dorsal frontoparietal network involved in planning (Przybylski and Króliczak, 2017), action emulation (Ptak et al., 2017), observation and execution of manipulative actions

544 (Dinstein, Hasson, Rubin, \& Heeger, 2007; Ferri, Rizzolatti, \& Orban, 2015; Lanzilotto et al., 2019; Orban, Ferri, \& Platonov, 2019; Reynaud, Lesourd, Navarro, \& Osiurak, 2016; Reynaud et al., 2019) and could also play a more general role in action understanding, and therefore in social interactions, by representing actor-object interactions at a higher level of abstraction (Tunik et al., 2007; Ramsey and Hamilton, 2010). Our results suggest that discriminating whether goal-directed actions have a social purpose is less efficient in IPS/SPL of adolescents and improves gradually, as indicated by the linear correlation between decoding accuracy and age in the adolescent group. point of view (reviewed in Burnett et al. 2011), it is perhaps not surprising to observe differences in the representation of the social dimension of actions. The lower decoding performance for the transitive dimension in adolescents in the pSTS is however less expected, considering that the understanding of object manipulation is certainly well mastered at this

557 age. Our data might thus indicate that action representation, at the perceptual level, subtending action categorization in the pSTS might still change in adolescence. It has to be noted however that, in the pSTS, the social/non-social actions discrimination accuracy was

560 weaker compared to transitive/intransitive actions and also not as high as in LOTC or 
IPS/SPL, like in Wurm and colleagues (2017) study; at individual level the decoding was significant (permutation tests) on only about half of the adults and one-third of the adolescents. It is coherent with the interpretation that pSTS responds to mutual interactions

564 between coacting agents (Isik et al., 2017; Walbrin et al., 2018): there was no mutual interactions between actors neither in Wurm and colleagues (2017) nor in our study (i.e., one acting agent and one passive agent). In any case the fact that we observed age differences for

567 both the social and the transitive dimensions indicates that the representation of action categories in this region is still different from that of adults. that different patterns of voxels may capture subtle changes between adolescents and adults that could not be revealed at the voxel-level. Differences in decoding accuracies between groups might be explained by different inter-subject variability (Bray et al., 2009). Individuals are maturing at different rates, and our adolescents' sample is likely more heterogeneous than versus non-social actions was decoded in only half of adolescents compared to $80 \%$ of adults

576 (see Extended data Figure 4-2). Yet this is not the case in the other regions where higher 577 decoding accuracy is observed in adults despite a similar proportion of adults and adolescents with significant decoding. This shows that interindividual variability in functional organization may account for only some but not all differences between adolescents and

580 adults and that inter-subject variability decreases with age non-homogeneously in different 581 AON parts. the vMPFC and TPJ, two regions commonly attributed to the mentalizing network (Frith and

584 Frith, 2007; Van Overwalle and Baetens, 2009), usually engaged when people make

585 attributions about the mental states of others. Developmental studies reported that during such 
tasks, adolescents activated the MPFC to a greater extent than adults (reviewed in Blakemore,

587 2008). It may be that during our task, adolescents also inferred thoughts and intentions, independently of the transitive or social nature of the actions. Future studies should investigate behavioral correlates of viewing these actions as well as links between the AON 590 and mentalizing areas.

592 adolescence. In line with our hypothesis, we revealed age differences in the local pattern of 593 activation representing the social dimension of an action in LOTC, IPS/SPL and pSTS, as

594 well as strengthening of the representation of the transitive dimension in the pSTS. We 595 observed no evidence of differences in the precentral regions. This underlies adolescent 596 development in the functional organization of the posterior parts of the AON. Future studies 597 should investigate how other featural or contextual components of actions are represented in 598 the AON of adolescents, in relation to changes in social perception skills.

\section{Data and Code availability statement}

602 Unthresholded statistical maps for the main contrasts of interest can be visualized on

603 NeuroVault (https://neurovault.org/collections/8403/). Behavioral and preprocessed

604 neuroimaging data will be posted on a public repository (OpenfMRI) after publication of the 605 research article. Stimulus materials and code are available upon reasonable request. 


\section{References}

608 Abraham A, Pedregosa F, Eickenberg M, Gervais P (2014) Machine learning for neuroimaging with scikit-learn. 8:1-10.

610 Becchio C, Cavallo A, Begliomini C, Sartori L, Feltrin G, Castiello U (2012) Social grasping:

611 From mirroring to mentalizing. Neuroimage 61:240-248

612 Behzadi Y, Restom K, Liau J, Liu TT (2007) A component based noise correction method 613 (CompCor) for BOLD and perfusion based fMRI. Neuroimage 37:90-101

614 Benjamini Y, Yekutieli D (2001) The control of the false discovery rate in multiple testing under dependency. Ann Stat 29:1165-1188.

Biagi L, Cioni G, Fogassi L, Guzzetta A, Sgandurra G, Tosetti M (2016) Action observation network in childhood: a comparative fMRI study with adults. Dev Sci 19:1075-1086.

Blakemore SJ (2008) The social brain in adolescence. Nat Rev Neurosci 9:267-277.

Bracci S, Daniels N, Op de Beeck H (2017) Task Context Overrules Object- and Category-

Bray S, Chang C, Hoeft F (2009) Applications of multivariate pattern classification analyses in developmental neuroimaging of healthy and clinical populations. Front Hum Neurosci 3

Buccino G, Binkofski F, Fink GR, Fadiga L, Fogassi L, Gallese V, Seitz RJ, Zilles K, Rizzolatti G, Freund J (2001) Action observation activates premotor and parietal areas in a somatotopic manner: an fMRI study. Eur J Neurosci 13:400-404.

628 Carr L, Iacoboni M, Dubeau M-C, Mazziotta JC, Lenzi GL (2003) Neural mechanisms of empathy in humans: a relay from neural systems for imitation to limbic areas. Proc Natl Acad Sci U S A 100:5497-5502.

631 Carter RM, Huettel SA (2013) A nexus model of the temporal-parietal junction. Trends Cogn 
Caspers S, Zilles K, Laird AR, Eickhoff SB (2010) ALE meta-analysis of action observation and imitation in the human brain. Neuroimage 50:1148-1167

Centelles L, Assaiante C, Nazarian B, Anton JL, Schmitz C (2011) Recruitment of both the mirror and the mentalizing networks when observing social interactions depicted by point-lights: A neuroimaging study. PLoS One 6:e15749.

Chen Q, Garcea FE, Mahon BZ (2016) The Representation of Object-Directed Action and Function Knowledge in the Human Brain. Cereb Cortex 26:1609-1618

Daprati E, Sirigu A (2006) How we interact with objects: learning from brain lesions. Trends Cogn Sci 10:265-270.

Dinstein I, Hasson U, Rubin N, Heeger DJ (2007) Brain Areas Selective for Both Observed and Executed Movements. J Neurophysiol 98:1415-1427

Downing PE (2001) A Cortical Area Selective for Visual Processing of the Human Body. Science (80 ) 293:2470-2473

Downing PE, Peelen M V. (2011) How might occipitotemporal body-selective regions interact with other brain areas to support person perception? Cogn Neurosci 2:216-226

Etzel JA, Braver TS (2013) MVPA permutation schemes: Permutation testing in the land of cross-validation. In: Proceedings - 2013 3rd International Workshop on Pattern Recognition in Neuroimaging, PRNI 2013, pp 140-143. IEEE.

Ferri S, Rizzolatti G, Orban GA (2015) The organization of the posterior parietal cortex devoted to upper limb actions: An fMRI study. Hum Brain Mapp 36:3845-3866.

653 Frith CD, Frith U (2007) Social Cognition in Humans. Curr Biol 17:724-732.

654 Gallese V, Fadiga L, Fogassi L, Rizzolatti G (1996) Action recognition in the premotor $655 \quad$ cortex. Brain 119:593-609.

656 Grosbras MH, Beaton S, Eickhoff SB (2012) Brain regions involved in human movement 
perception: A quantitative voxel-based meta-analysis. Hum Brain Mapp 33:431-454.

Haynes JD (2015) A Primer on Pattern-Based Approaches to fMRI: Principles, Pitfalls, and Perspectives. Neuron 87:257-270 AP (2004) Watching social interactions produces dorsomedial prefrontal and medial parietal BOLD fMRI signal increases compared to a resting baseline. Neuroimage 21:1167-1173.

664

Isik L, Koldewyn K, Beeler D, Kanwisher N (2017) Perceiving social interactions in the posterior superior temporal sulcus. Proc Natl Acad Sci 114:E9145-E9152

Kao MH, Mandal A, Lazar N, Stufken J (2009) Multi-objective optimal experimental designs for event-related fMRI studies. Neuroimage 44:849-856

Kasper L, Bollmann S, Diaconescu AO, Hutton C, Heinzle J, Iglesias S, Hauser TU, Sebold M, Manjaly Z, Pruessmann KP, Stephan KE (2017) The PhysIO Toolbox for Modeling Physiological Noise in fMRI Data. J Neurosci Methods 276:56-72

Lloyd-Fox S, Blasi A, Volein A, Everdell N, Elwell CE, Johnson MH (2009) Social Perception in Infancy: A Near Infrared Spectroscopy Study. Child Dev 80:986-999

678 Mahon B, Milleville S, Negri GA, Rumiati R, Caramazza A, Martin A (2007) Action-Related 
682

683

684

685

686

687

688

689

690

691

692

693

694

695

696

697

698

699

700

701

702

703

704

705

706

Marshall WA, Tanner M (1970) Variations in the pattern of pubertal changes in boy. Arch Dis Child 45:13-23.

Mills KL, Lalonde F, Clasen LS, Giedd JN, Blakemore SJ (2014) Developmental changes in the structure of the social brain in late childhood and adolescence. Soc Cogn Affect Neurosci 9:123-131.

Montgomery KJ, Isenberg N, Haxby J V. (2007) Communicative hand gestures and objectdirected hand movements activated the mirror neuron system. Soc Cogn Affect Neurosci $2: 114-122$

Morales S, Bowman LC, Velnoskey KR, Fox NA, Redcay E (2019) An fMRI study of action observation and action execution in childhood. Dev Cogn Neurosci 37:100655

Oberman LM, Pineda JA, Ramachandran VS (2007) The human mirror neuron system: A link between action observation and social skills. Soc Cogn Affect Neurosci 2:62-66

Ohnishi T, Moriguchi Y, Matsuda H, Mori T, Hirakata M, Imabayashi E, Hirao K, Nemoto K, Kaga M, Inagaki M, Yamada M, Uno A (2004) The neural network for the mirror system and mentalizing in normally developed children: An fMRI study. Neuroreport 15:14831487.

Orban GA, Ferri S, Platonov A (2019) The role of putative human anterior intraparietal sulcus area in observed manipulative action discrimination. Brain Behav 9:e01226

Petersen AC, Crockett L, Richards M, Boxer A (1988) A self-report measure of pubertal status: Reliability, validity, and initial norms. J Youth Adolesc 17:117-133

Pokorny JJ, Hatt N V., Colombi C, Vivanti G, Rogers SJ, Rivera SM (2015) The Action Observation System when Observing Hand Actions in Autism and Typical Development. Autism Res 8:284-296

Przybylski Ł, Króliczak G (2017) Planning Functional Grasps of Simple Tools Invokes the Hand-independent Praxis Representation Network: An fMRI Study. J Int Neuropsychol 
Soc 23:108-120

Ptak R, Schnider A, Fellrath J (2017) The Dorsal Frontoparietal Network: A Core System for Emulated Action. Trends Cogn Sci 21:589-599

Ramsey R, Hamilton AF d. C (2010) Triangles have goals too: Understanding action representation in left aIPS. Neuropsychologia 48:2773-2776

Reynaud E, Lesourd M, Navarro J, Osiurak F (2016) On the neurocognitive origins of human tool use: A critical review of neuroimaging data. Neurosci Biobehav Rev 64:421-437.

Reynaud E, Navarro J, Lesourd M, Osiurak F (2019) To Watch is to Work: a Review of NeuroImaging Data on Tool Use Observation Network. Neuropsychol Rev 29:484-497

Ross PD, de Gelder B, Crabbe F, Grosbras M-H (2014) Body-selective areas in the visual cortex are less active in children than in adults. Front Hum Neurosci 8:941

Saggar M, Shelly EW, Lepage JF, Hoeft F, Reiss AL (2014) Revealing the neural networks associated with processing of natural social interaction and the related effects of actororientation and face-visibility. Neuroimage 84:656-648

Scherf KS, Behrmann M, Humphreys K, Luna B (2007) Visual category-selectivity for faces, places and objects emerges along different developmental trajectories. Dev Sci 10:15-30

Shaw DJ, Grosbras M-H, Leonard G, Pike GB, Paus T (2011) Development of Functional Connectivity during Adolescence: A Longitudinal Study Using an Action-Observation Paradigm. J Cogn Neurosci 23:3713-3724

Shaw DJ, Grosbras MH, Leonard G, Pike GB, Paus T (2012) Development of the action observation network during early adolescence: A longitudinal study. Soc Cogn Affect Neurosci 7:64-80.

Shimada S, Hiraki K (2006) Infant's brain responses to live and televised action. Neuroimage 32:930-939

Sliwa J, Freiwald WA (2017) A dedicated network for social interaction processing in the 
Steinberg L, Morris AS (2001) Adolescent Development. Annu Rev Psychol 52:83-110

Tunik E, Rice NJ, Hamilton A, Grafton ST (2007) Beyond grasping: representation of action in human anterior intraparietal sulcus. Neuroimage 36:77-86.

Van Essen DC (2005) A Population-Average, Landmark- and Surface-based (PALS) atlas of human cerebral cortex. Neuroimage 28:635-662.

Van Overwalle F, Baetens K (2009) Understanding others' actions and goals by mirror and mentalizing systems: A meta-analysis. Neuroimage 48:564-584.

Wager TD, Nichols TE (2003) Optimization of experimental design in fMRI: A general framework using a genetic algorithm. Neuroimage 18:293-309.

Wagner DD, Kelley WM, Haxby J V., Heatherton TF (2016) The dorsal medial prefrontal cortex responds preferentially to social interactions during natural viewing. J Neurosci $36: 6917-6925$.

Walbrin J, Downing P, Koldewyn K (2018) Neural responses to visually observed social interactions. Neuropsychologia 112:31-39

Wurm MF, Ariani G, Greenlee MW, Lingnau A (2016) Decoding Concrete and Abstract Action Representations During Explicit and Implicit Conceptual Processing. Cereb Cortex 26:3390-3401

Wurm MF, Caramazza A (2019) Lateral occipitotemporal cortex encodes perceptual components of social actions rather than abstract representations of sociality. Neuroimage 202:116153

Wurm MF, Caramazza A, Lingnau A (2017) Action Categories in Lateral Occipitotemporal Cortex Are Organized Along Sociality and Transitivity. J Neurosci 37:562-575 
$758 \quad$ Figures

759 Figure 1. (A) stimuli used in the present study varying across two dimensions: sociality

760 (social, non-social) and transitivity (transitive, intransitive), leading to 4 distinct categories of actions: Social Transitive (ST), Non-Social Transitive (NT), Social Intransitive (SI), , and

762 Non-Social Intransitive (NI). Each category was made of four classes of actions: (ST): Give:

763 the actor moves a book or a pen from his/her peri-personal space toward the peri-personal

764 space of the passive actor; Take: the reverse of Give; (NT): Open: the actor opens a

765 notebook; Close: the reverse of Open ; Rub: the actor rubs pencil trace on the notebook with

766 rapid oscillatory movements; Write: the actor writes something on the notebook with the

767 pencil; (SI) Agree: the actor indicates with a gesture (i.e., thumb up) to the passive actor that

768 he agrees; Disagree: the actor indicates to the passive actor with a gesture (i.e., thumb down)

769 that he disagrees; Come: the actor indicates with his active hand to the passive actor to come

770 closer; Go away: the reverse of Come; (NI): Stroke: the actor strokes his forearm with his

771 active hand; Scratch: the actor scratches his forearm with his active hand. (B) schematic

772 depiction of the sequence of events in a representative session.

774 Figure 2. Schematic representation of the MVPA. A beta estimate was first extracted for each

775 trial using a GLM. The SVM classification was performed using a leave-one-out cross-

776 validation scheme. A SVM classifier was trained $(112 \beta)$ and tested to discriminate between

777 Social versus Non-Social actions (16 $\beta)$ and between Transitive versus Intransitive (16 $\beta$ )

778 actions. Classification accuracies were averaged across iterations ( 8 iterations) and entered in

779 a two-way ANOVA with Age group (Adolescents and Adults) as between factor and 
hemisphere (Left and Right) as within factor, for each ROI and each Action Dimension separately.

Figure 3. Brain activation associated with main effect of (A) Sociality; (B) Transitivity; and (3) Group. Activations are projected on PALS-B12 atlas surface configurations (Van Essen, 2005) : lateral fiducial surfaces. Statistical maps are FWE-corrected for multiple comparisons across the whole-brain at the cluster level; FWE, $p<.05)$. AIPS: anterior intraparietal sulcus; SPL: superior parietal lobe; pSTS: posterior superior temporal sulcus; MTG: middle temporal gyrus; STS: superior temporal sulcus; iLOC: inferior lateral occipital cortex; Occ fusif G: occipital fusiform gyrus; Intracal: intracalcarine cortex; SMG: supramarginal gyrus; AG: angular gyrus; PostG: postcentral gyrus; dPMC: dorsal premotor cortex; LOC: lateral occipital cortex; TP: temporal pole; TOf: temporo-occipital fusiform gyrus; Lingual G: Lingual gyrus; EBA: extrastriate body area; FBA: fusiform body area; EVC: extrastriate visual cortex; vMPFC: ventral medial prefrontal cortex; TPJ: temporoparietal junction.

Figure 4. ROI MVPA results. (A) Illustration of the 8 functionally defined ROIs used in the present study derived from the meta-analysis of Grosbras et al. (2012). MNI-coordinates of the different ROIs are represented in Extended Data Table 4-1. (B) Group averaged decoding accuracies for (B) decoding social versus non-social (blue) and (C) transitive versus intransitive (red) actions for adolescents (dark) and adults (light). Error bars indicate Standard

800 Deviation (SD). Asterisk represents statistical significance (FDR-corrected for the number of 801 tests). Dotted line indicates decoding accuracy at chance-level (50\%). Individual data is 
804 Figure 5. MVPA searchlight analyses. (A) Mean accuracy maps and statistical comparison

805 maps of the searchlight decoding for Social versus Non-Social actions ( chance level $=50 \%$ )

806 and for Transitive versus Intransitive actions (chance level $=50 \%$ ) for Adults and

807 Adolescents. (B) Comparison of Searchlight accuracy maps of Adults and Adolescents using

808 two-sample t-tests for Sociality and Transitivity separately. Corrections for multiple

809 comparisons were applied at the cluster level (FWE, $p<.05)$. Coordinates of significant

810 clusters are presented in Extended data Table 5-1.

811

812 Figure 6. Confusion matrices for each action class in ROIs for adolescents and adults,

813 providing the percentage of correct classifications (diagonals) and misclassifications (off

814 diagonals). The lower the percentage, the more the cell is light-yellow colored and the higher

815 the percentage, the more the cell is dark blue colored. Comparison of diagonal values to

816 chance (0.5) are presented on Extended data Figure 6-1.

818 Figure 7. Mean decoding accuracies in IPS/SPL for each category of action for adolescents

819 (dark) and adults (light). Upper panel: ANOVA on mean decoding accuracies with sociality 820 and transitivity as within-subject factors and Age group as between-subject factor. Bottom

821 panels: mean decoding accuracies are plotted against chronological age for each group and

822 each class action (NI, NT, SI, and ST). Significant coefficient correlations (Pearson) are

823 indicated in red.

824 Dotted lines represent decoding accuracy at chance-level $(25 \%){ }^{*} p<.05,{ }^{* *} p<.01$. 


\section{Figure 1.}

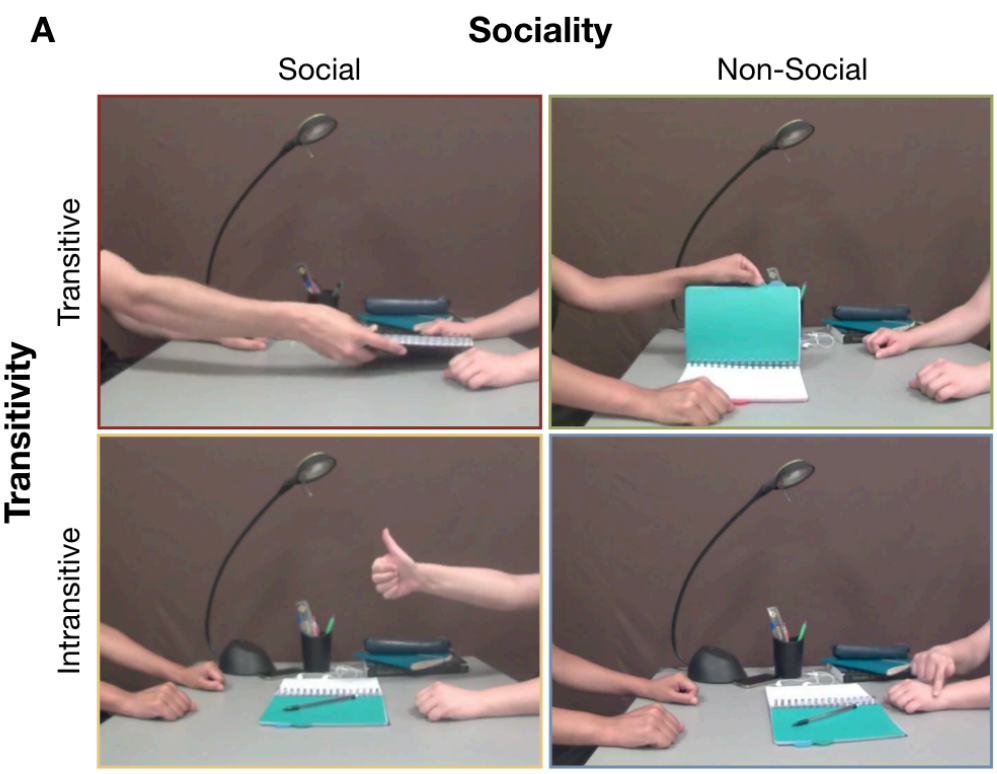

B

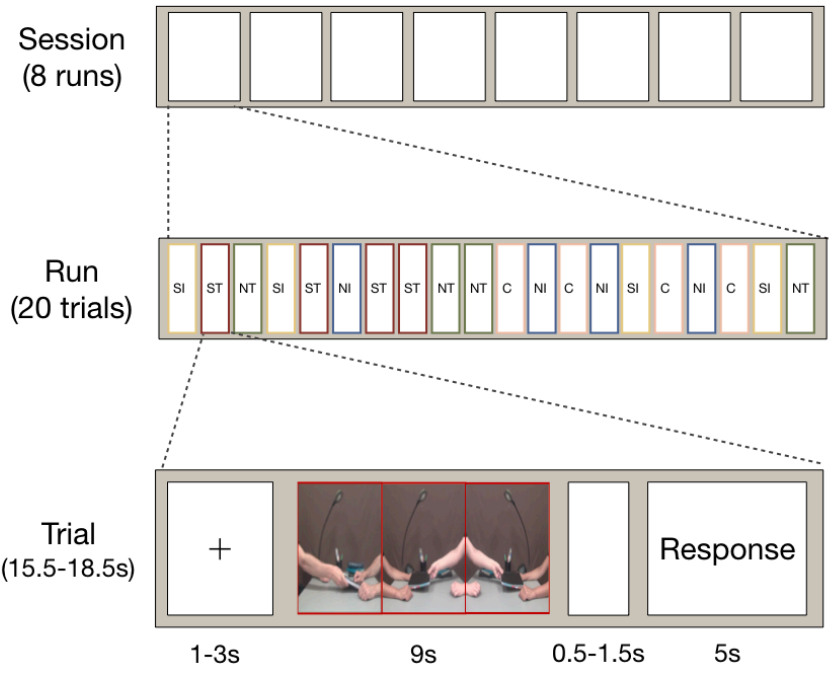

829 


\section{$830 \quad$ Figure 2.}

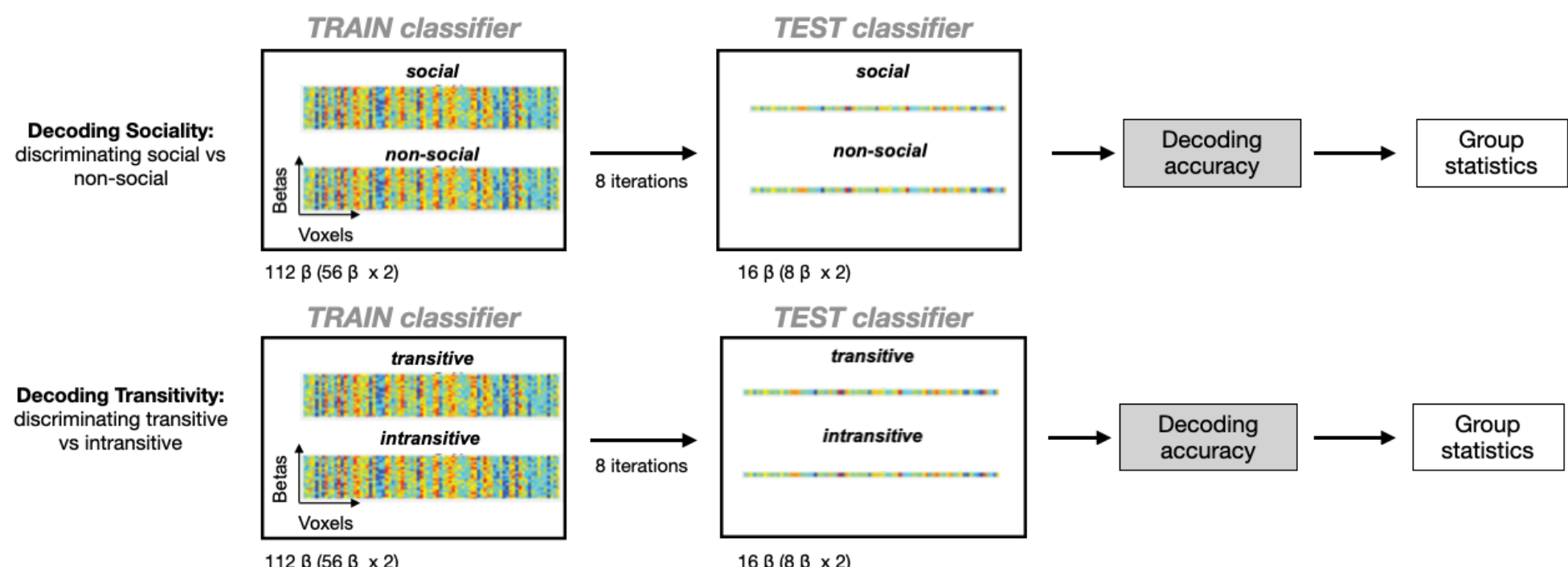

832 
bioRxiv preprint doi: https://doi.org/10.1101/2020.1129.402560; this version posted November 29, 2020. The copyright holder for this preprint (which was not certified by peer review) is the author/funder. All rights reserved. No reuse allowed without permission.

\section{$833 \quad$ Figure 3.}

\section{A. Main effect of Sociality}

Left
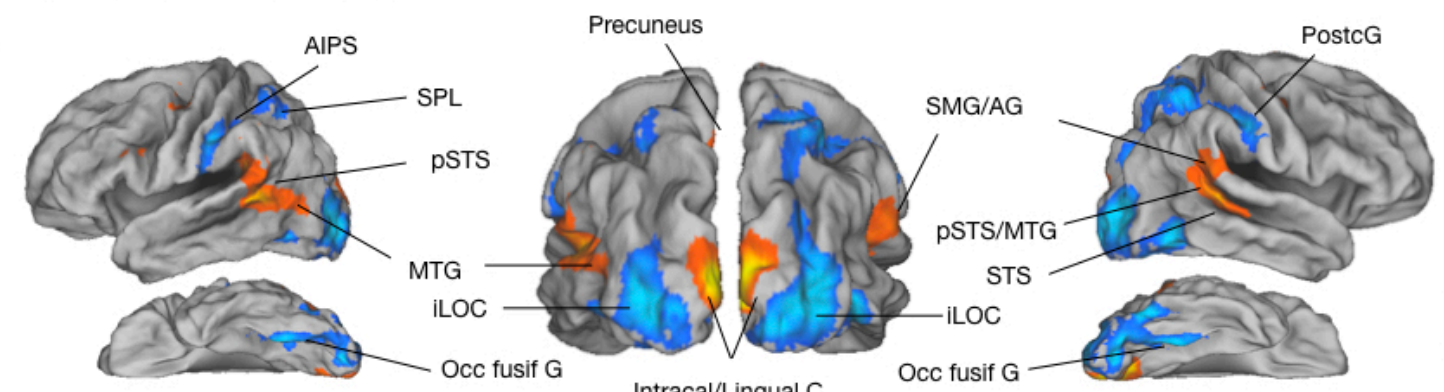

Right

B. Main effect of Transitivity
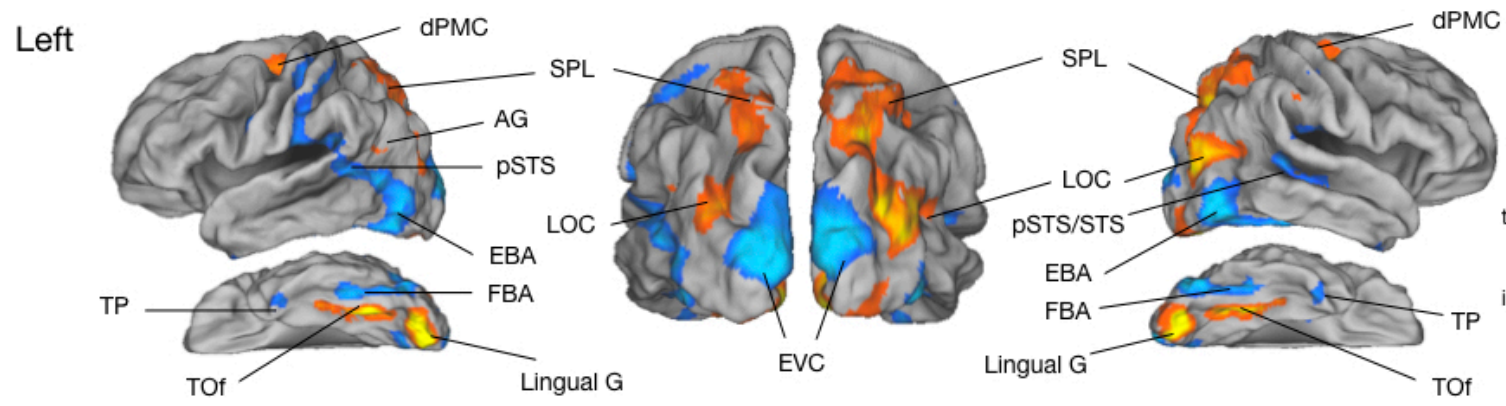

Right

C. Main effect of Group

Left

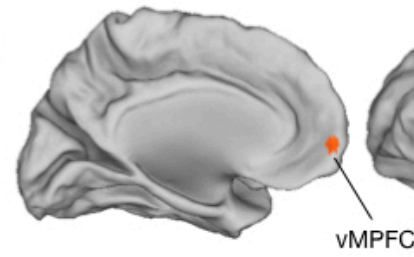

8
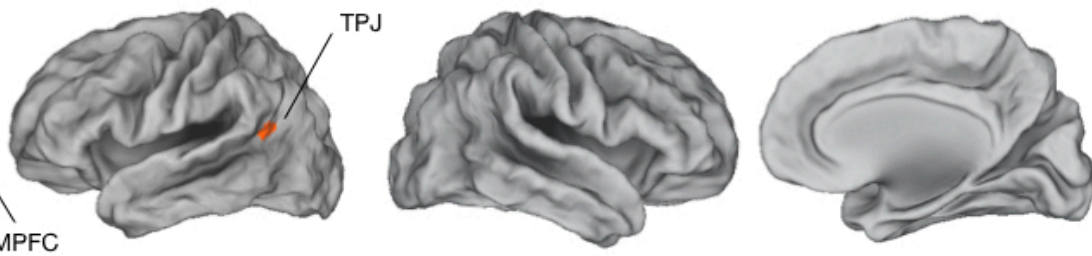

Right

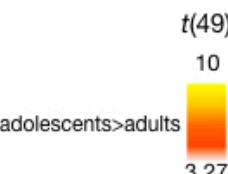

835 


\section{$836 \quad$ Figure 4}

\section{Regions of interest location}

A.

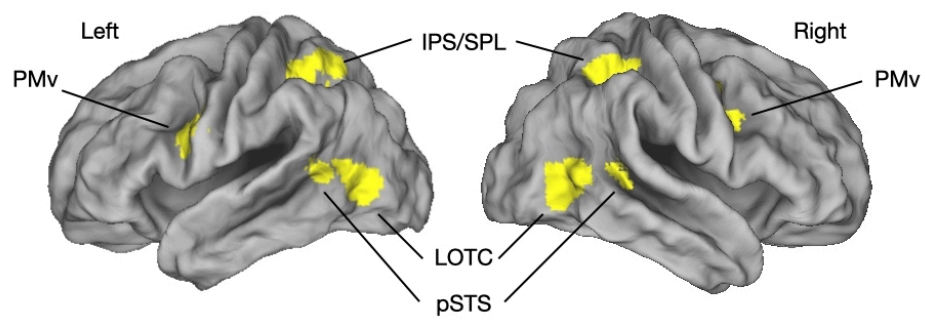

B.

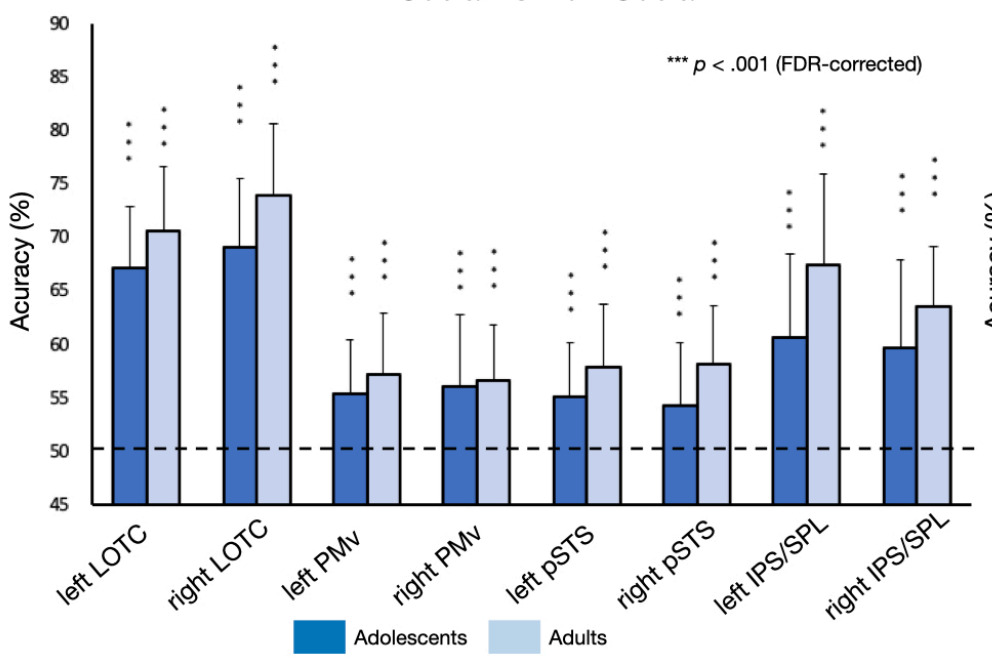

C.

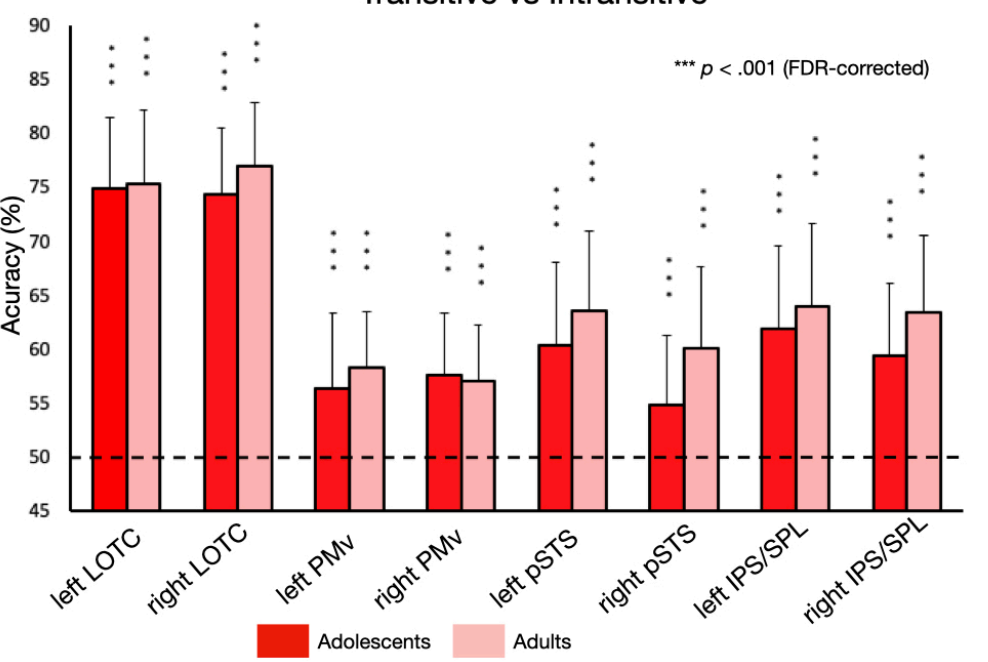

838 
bioRxiv preprint doi: https://doi.org/10.1101/2020.11.29.402560; this version posted November 29, 2020. The copyright holder for this preprint (which was not certified by peer review) is the author/funder. All rights reserved. No reuse allowed without permission.

840

A. Adolescents
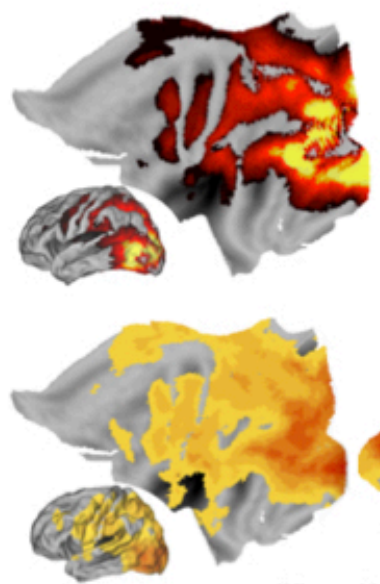

$t(26), p<.05$ (FWE)
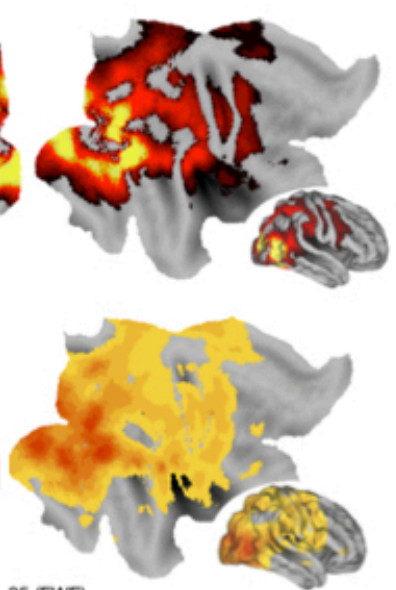

28

841

B.

\section{Sociality}

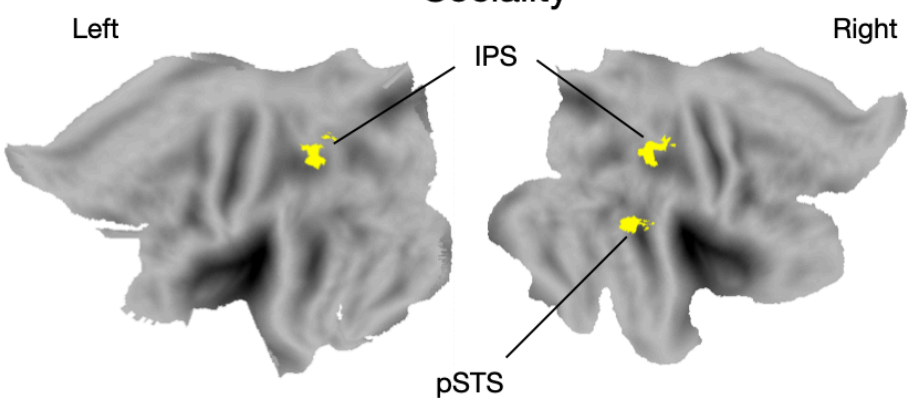

Adolescents
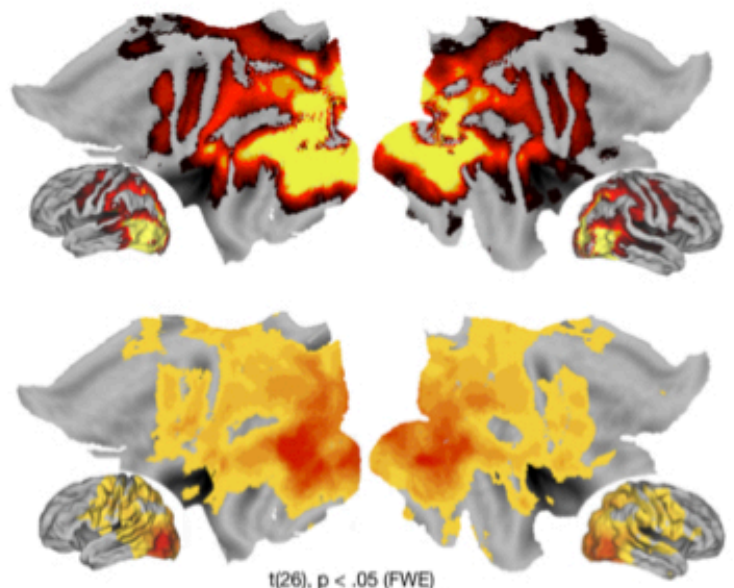

28

\section{Sociality}

Adults
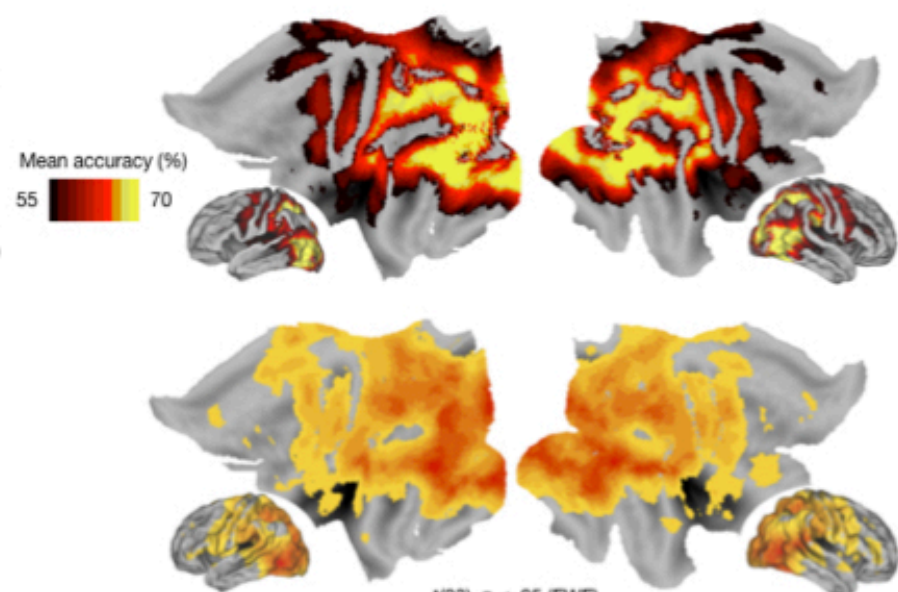

(23), $p<.05$ (FWE)

028

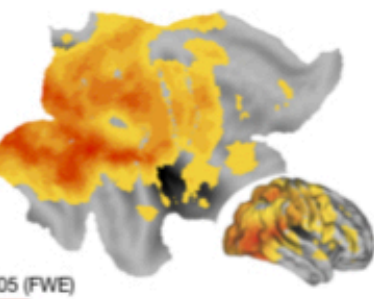

\section{Transitivity}

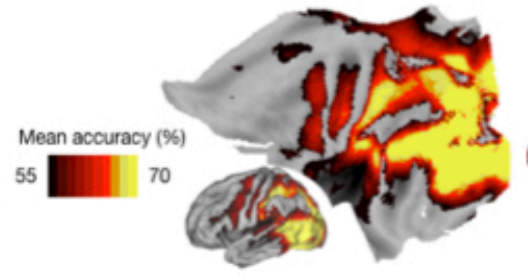

Adults

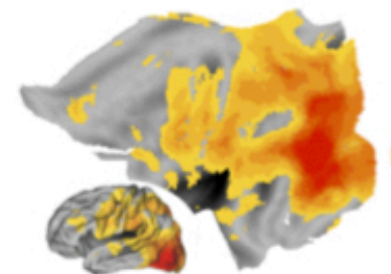

t(23), $p<.05$ (FWE)

$0 \quad 28$

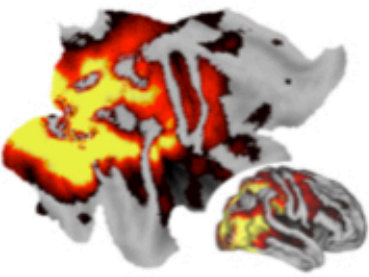

Transitivity

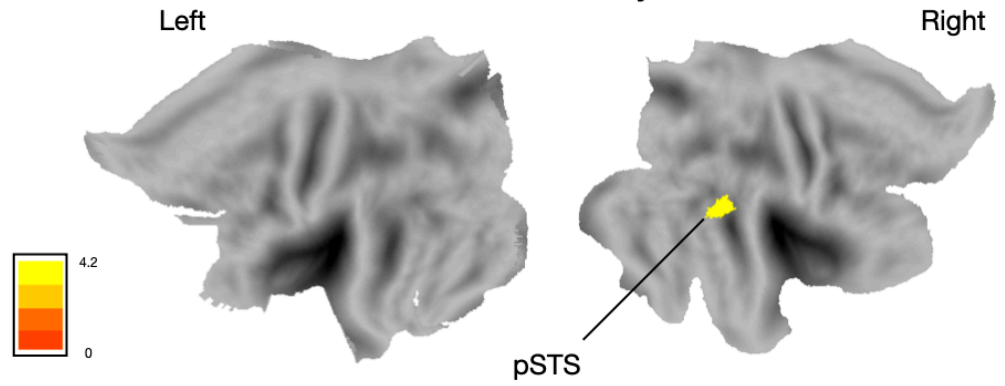




\section{$843 \quad$ Figure 6}

844

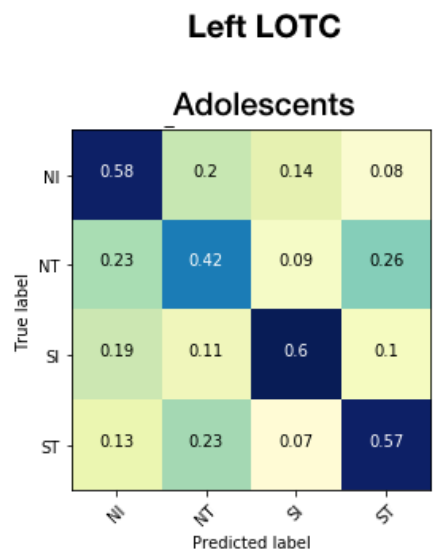

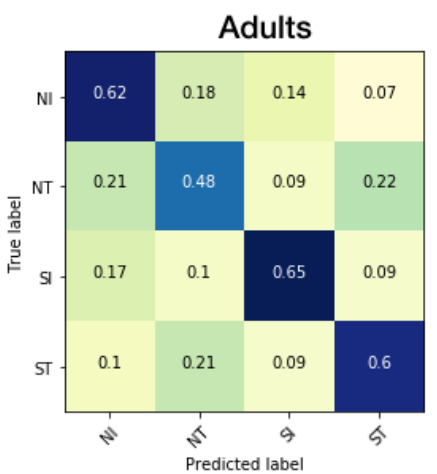

left pSTS
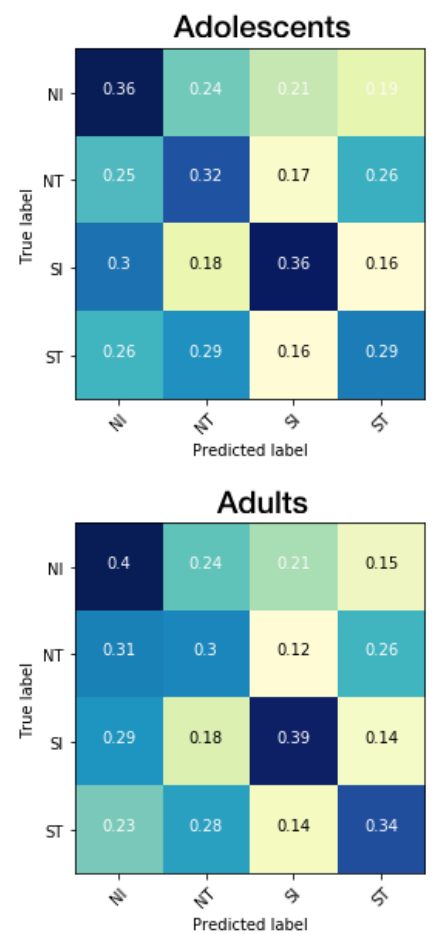
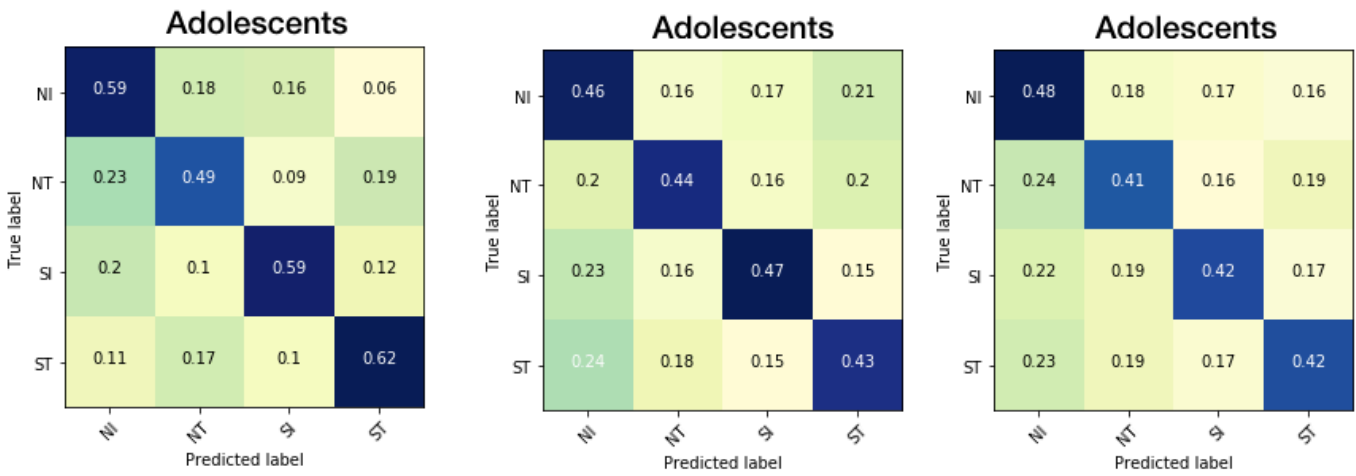

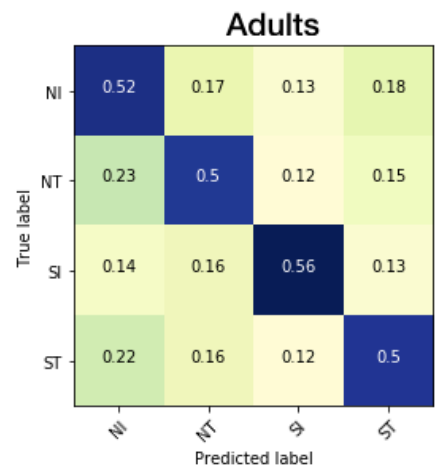

Left PMv

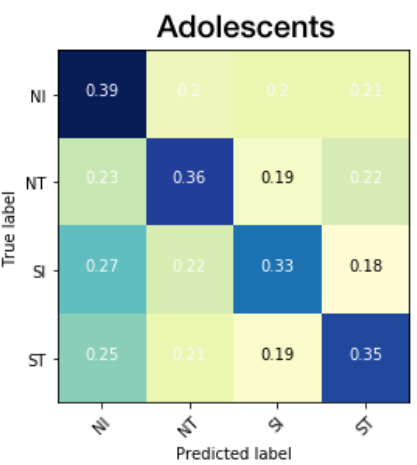

Right IPS

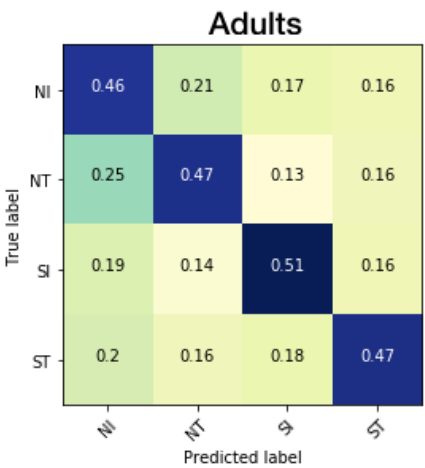

Right PMv

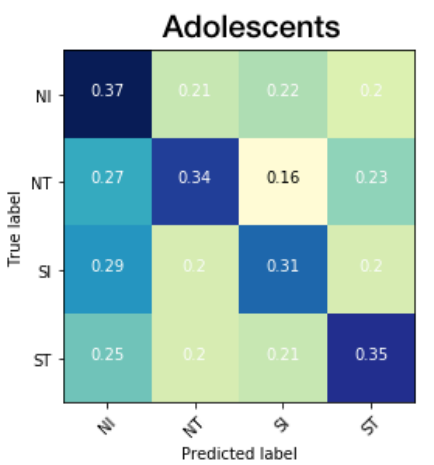

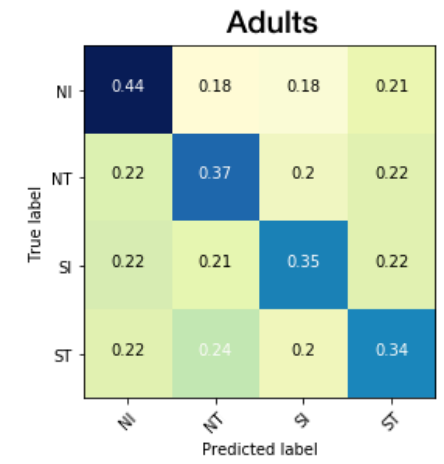
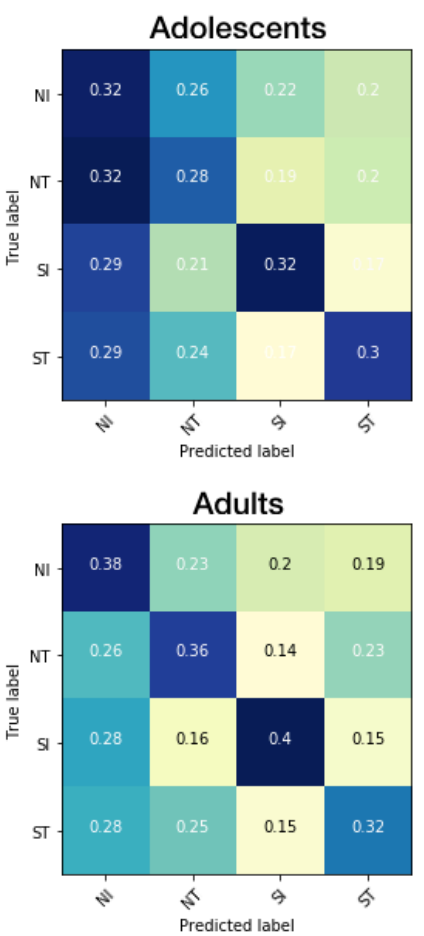

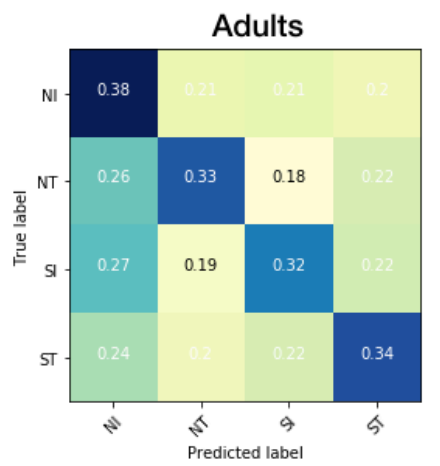

845 


\section{$846 \quad$ Figure 7}

847

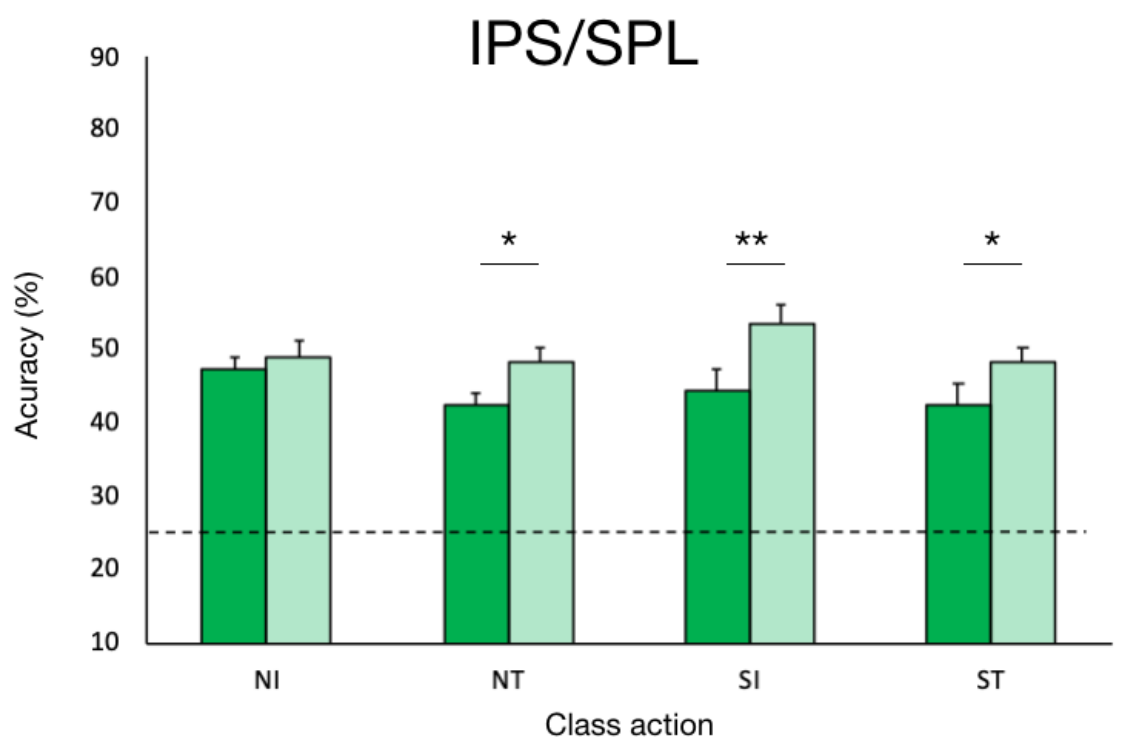

848

NI
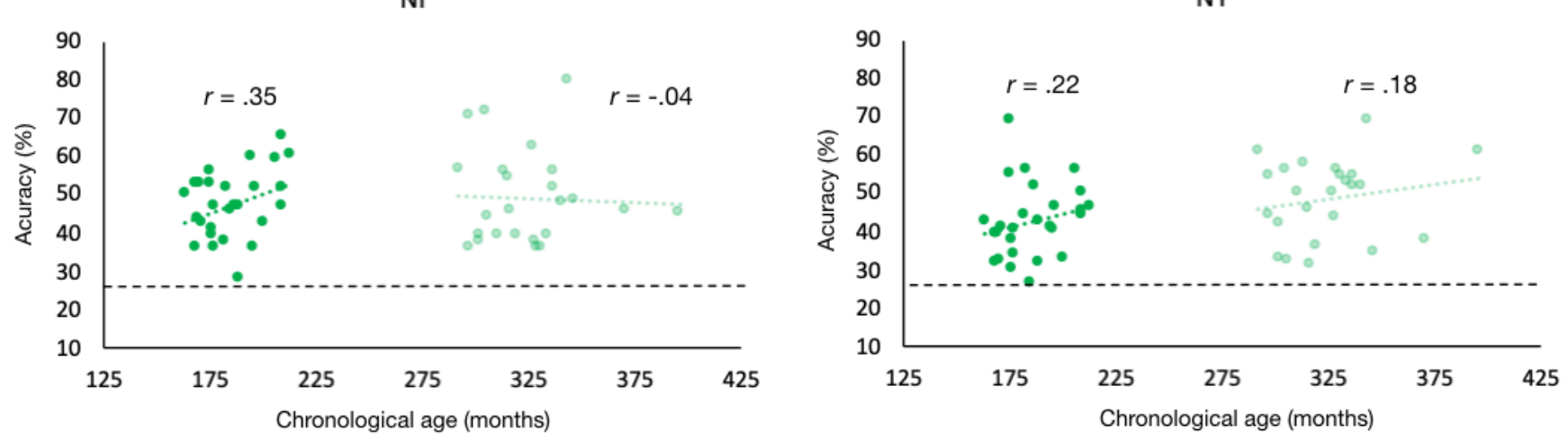

SI

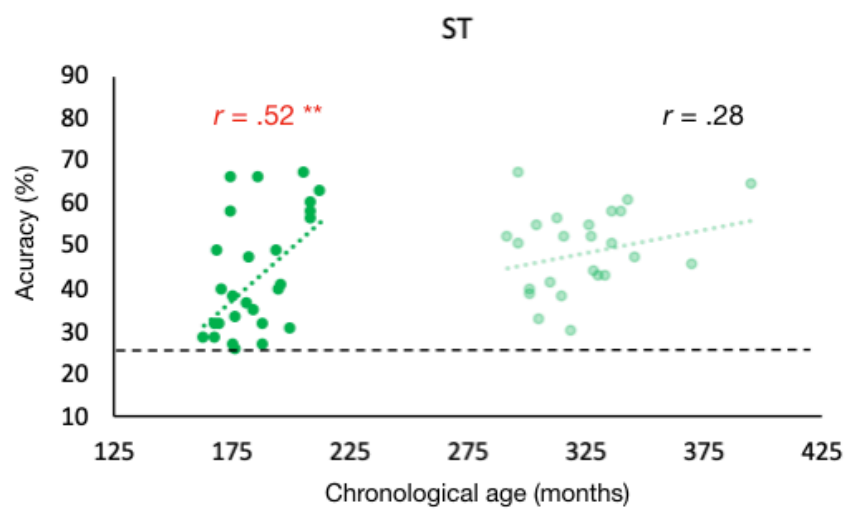

850 
Table 1. Brain regions activated in the whole-brain analysis for the main effect of Age Group, Sociality and Transitivity

\begin{tabular}{|c|c|c|c|c|c|c|}
\hline \multicolumn{2}{|l|}{ Region Label } & \multirow[t]{2}{*}{ Extent } & \multirow[t]{2}{*}{ t-value } & \multicolumn{3}{|c|}{ Peak MNI Coordinates } \\
\hline \multicolumn{5}{|l|}{ Main effect of Group } & & \\
\hline \multicolumn{7}{|l|}{ Adolescents $>$ adults } \\
\hline Ventral Medial Prefrontal Cortex & $\mathrm{L}$ & 58 & 5.94 & -6 & 61 & -8 \\
\hline Temporo Parietal Junction & $\mathrm{L}$ & 116 & 4.49 & -43 & -59 & 25 \\
\hline \multicolumn{7}{|l|}{ Main effect of Sociality } \\
\hline \multicolumn{7}{|l|}{ Social/Non-Social } \\
\hline Visual cortex & $\mathrm{L} / \mathrm{R}$ & 8217 & & & & \\
\hline Intracalcarine Cortex & & & 11.29 & 7 & -79 & 3 \\
\hline Intracalcarine Cortex & & & 10.93 & -8 & -97 & 13 \\
\hline Lingual Gyrus & & & 9.52 & -3 & -79 & -10 \\
\hline Temporo-parietal Cortex & $\mathrm{L}$ & 1047 & & & & \\
\hline pSTS/Middle Temporal Gyrus & & & 8.47 & -53 & -47 & 8 \\
\hline Supramarginal Gyrus & & & 7.48 & -51 & -42 & 25 \\
\hline Angular Gyrus & & & 6.95 & -56 & -62 & 10 \\
\hline Temporo -parietal & $\mathrm{R}$ & 749 & & & & \\
\hline $\mathrm{pSTS} / \mathrm{MTG}$ & & & 7.97 & 47 & -42 & 10 \\
\hline Supramarginal Gyrus & & & 6.33 & 67 & -39 & 23 \\
\hline STS middle & & & 6.03 & 50 & -32 & -3 \\
\hline Precuneus & $\mathrm{L} / \mathrm{R}$ & 373 & 6.19 & -1 & -52 & 58 \\
\hline Precentral Gyrus & $\mathrm{L}$ & 508 & 6.15 & -41 & -7 & 53 \\
\hline Superior Frontal Gyrus & & & 5.02 & -26 & 4 & 60 \\
\hline Pre-SMA & & & 4.49 & 12 & -4 & 63 \\
\hline Precentral Gyrus & $\mathrm{R}$ & 114 & 5.69 & 47 & 1 & 55 \\
\hline
\end{tabular}


Superior Parietal Lobule Inferior Frontal Gyrus

\section{Non-Social/Social}

Visual cortex

Occipital Pole / Lateral Occipital

Occipital Pole / Lateral Occipital

Occipital fusiform gyrus

Temporal Occipital Fusiform Cortex

Anterior parietal cortex Postcentral Gyrus/AIPS

Central Opercular Cortex

Superior Parietal Lobule

inferior Lateral Occipital Cortex

Precentral Gyrus

\section{Main effect of Transitivity}

\section{Transitive / Intransitive}

Medial occipital cortex

Lingual gyrus

Lingual gyrus

Temporal Occipital Fusiform

Temporal Occipital Fusiform

Precentral Cortex

Precentral cortex

Superior Frontal sulcus

Parieto-occipital Cortex

Lateral occipital

Superior Parietal

Inferior temporal Cortex

Cerebellum (lobule VIIII/ IX)

$\begin{array}{cccccc}\mathrm{L} & 78 & 4.97 & -33 & -49 & 35 \\ \mathrm{~L} & 55 & 4.37 & -46 & 14 & 23 \\ \mathrm{~L} / \mathrm{R} & 8217 & & & & \\ & & 12.61 & 32 & -92 & 5 \\ & & 12.45 & -28 & -89 & 0 \\ & & 12.35 & 17 & -87 & -8 \\ & & 11.29 & 27 & -49 & -18 \\ \mathrm{~L} & 900 & & & & \\ & & 8.97 & -51 & -22 & 33 \\ & & 6.62 & -56 & -17 & 18 \\ & & 6.45 & -28 & -47 & 68 \\ \mathrm{~L} & 92 & 7.55 & -46 & -69 & -8 \\ \mathrm{R} & 76 & 5.04 & 30 & -12 & 58\end{array}$

$\begin{array}{cccccc}\mathrm{L} / \mathrm{R} & 10781 & & & & \\ & & 16.50 & 15 & -87 & -10 \\ & & 15.30 & -8 & -89 & -10 \\ & & 13.37 & 30 & -52 & -13 \\ & & 12.53 & -27 & -55 & -16 \\ \mathrm{R} & 450 & 10.23 & 25 & -7 & 53 \\ \mathrm{~L} & 300 & 7.30 & -23 & 1 & 55 \\ \mathrm{R} & 74 & 5.70 & 22 & 21 & 40 \\ \mathrm{~L} & 929 & & & & \\ & & 9.97 & -33 & -82 & 20 \\ & & 7.45 & -28 & -52 & 65 \\ \mathrm{R} & 53 & 5.56 & 52 & -52 & -10 \\ \mathrm{R} & 83 & 5.45 & 15 & -47 & -50\end{array}$


Cerebellum (lobule VIIII/ IX)

Angular Gyrus

Posterior Cingulate Gyrus

\section{Intransitive $>$ Transitive}

Medial occipital (early visual) cortex

Cuneus

Cuneus

Intracalcarine Cortex

Lateral Occipital temporal cortex Inferior Lateral Occipital Cortex (EBA)

Temporal Occipital Fusiform Cortex (FBA)

Posterior Superior Temporal Cortex Supramarginal Gyrus

Post Superior Temporal Gyrus

Temporal pole

Temporal pole

Pericentral cortex

Central sulcus (hand area)

Central sulcus (index finger area)

$\begin{array}{cccccc}\mathrm{L} & 179 & 8.35 & -13 & -49 & -50 \\ \mathrm{~L} & 57 & 4.68 & -48 & -62 & 23 \\ \mathrm{R} & 92 & 4.53 & 12 & -29 & 43\end{array}$

L/R $\quad 10781$

$\begin{array}{llll}14.16 & 12 & -94 & 18\end{array}$

$\begin{array}{llll}12.65 & -11 & -99 & 8\end{array}$

$\begin{array}{llll}9.92 & -3 & -77 & 10\end{array}$

R $\quad 766$

$\begin{array}{llll}11.29 & 45 & -79 & -8\end{array}$

$\begin{array}{llll}7.50 & 45 & -44 & -20\end{array}$

R $\quad 527$

$\begin{array}{llll}7.09 & 52 & -37 & 8\end{array}$

$\begin{array}{llll}5.12 & 52 & -19 & -5\end{array}$

$\begin{array}{llll}6.61 & 37 & -4 & -45\end{array}$

$\begin{array}{llll}5.46 & -38 & -4 & -45\end{array}$

L $\quad 65$

R 313

3

\begin{tabular}{llll}
5.28 & 35 & -19 & 40 \\
4.68 & 40 & -24 & 60 \\
4.59 & 55 & -14 & 50 \\
\hline
\end{tabular}

All results are thresholded at $p<.05$ (FWE corrected for multiple comparisons at the cluster level) 